\title{
Enhanced Electron-Phonon Interaction in Multivalley Materials
}

\author{
Thibault Sohier, ${ }^{1, *}$ Evgeniy Ponomarev, $, 2,,{ }^{*}$ Marco Gibertini, ${ }^{2,1, \dagger}$ Helmuth Berger, ${ }^{4}$ \\ Nicola Marzari, ${ }^{1}$ Nicolas Ubrig, ${ }^{2,3}$ and Alberto F. Morpurgo ${ }^{2,3, \sharp}$ \\ ${ }^{1}$ Theory and Simulation of Materials (THEOS), and National Centre for Computational Design \\ and Discovery of Novel Materials (MARVEL), École Polytechnique Fédérale de Lausanne, \\ CH-1015 Lausanne, Switzerland \\ ${ }^{2}$ Department of Quantum Matter Physics, University of Geneva, \\ 24 Quai Ernest Ansermet, CH-1211 Geneva, Switzerland \\ ${ }^{3}$ Group of Applied Physics, University of Geneva, \\ 24 Quai Ernest Ansermet, CH-1211 Geneva, Switzerland \\ ${ }^{4}$ Laboratoire de Physique de la Matiére Complexe (LPMC), École Polytechnique Fédérale de Lausanne, \\ CH-1015 Lausanne, Switzerland
}

(Received 22 January 2019; revised manuscript received 30 May 2019; published 6 August 2019)

We report a combined experimental and theoretical investigation that reveals a new mechanism responsible for the enhancement of electron-phonon coupling in doped semiconductors in which multiple inequivalent valleys are simultaneously populated. Using Raman spectroscopy on ionic-liquid-gated monolayer and bilayer $\mathrm{MoS}_{2}, \mathrm{WS}_{2}$, and $\mathrm{WSe}_{2}$ over a wide range of electron and hole densities, we find that phonons with a dominant out-of-plane character exhibit strong softening upon electron accumulation while remaining unaffected upon hole doping. This unexpected-but very pronounced-electron-hole asymmetry is systematically observed in all monolayers and bilayers. By performing first-principles simulations, we show that the phonon softening occurs when multiple inequivalent valleys are populated simultaneously. Accordingly, the observed electron-hole asymmetry originates from the much larger energy separation between valleys in the valence bands-as compared to the conduction band-that prevents the population of multiple valleys upon hole accumulation. We infer that the enhancement of the electron-phonon coupling occurs because the population of multiple valleys acts to strongly reduce the efficiency of electrostatic screening for those phonon modes that cause the energy of the inequivalent valleys to oscillate out of phase. This robust mechanism is likely to play an important role in several physical phenomena, possibly including the occurrence of superconductivity in different transition metal dichalcogenides.

DOI: 10.1103/PhysRevX.9.031019

\section{INTRODUCTION}

The electronic and elastic properties of solids are determined by the interaction between charge carriers and the elementary excitations associated with the vibration of the crystalline lattice, namely, the phonons. In view of its fundamental relevance, the nature of the interaction between electrons and phonons has been investigated in great depth in many different contexts [1-3]. In the simplest possible terms, electron-phonon interaction can be

\footnotetext{
*These authors have contributed equally.

†marco.gibertini@unige.ch

*alberto.morpurgo@unige.ch
}

Published by the American Physical Society under the terms of the Creative Commons Attribution 4.0 International license. Further distribution of this work must maintain attribution to the author(s) and the published article's title, journal citation, and DOI.
Subject Areas: Condensed Matter Physics

understood as originating from the electrostatic potential generated by the lattice distortion associated with the ionic displacement in the presence of a phonon. In other words, exciting a phonon in a crystal displaces the charged ions from their equilibrium positions, thereby generating a local charge imbalance and a corresponding electrostatic potential (known as the deformation potential $[4,5]$ ), which in turn directly acts on all mobile electrons.

Depending on the specific type of material considered and on the level of detail needed for a precise microscopic understanding, the situation can be more complex. For instance, in some systems (including graphene), the coupling between the lattice deformation associated with certain phonon modes and charge carriers is described in terms of a vector potential, rather than an electrostatic one $[6,7]$. In these cases, the phonon perturbation of the electronic Hamiltonian is formally equivalent to a magnetic field [8]. Also, the influence of the electron-phonon interaction on physical phenomena depends strongly on 
whether the energy and momentum relaxation of electrons is fast or slow relative to the phonon frequency, corresponding to the so-called adiabatic or anti-adiabatic limit of electron-phonon coupling (EPC) $[9,10]$.

Irrespective of the specific situation, what is key to understanding electron-phonon interaction (and its strength) is electrostatic screening [3]. Indeed, the deformation potential generated by the excitation of a long-wavelength phonon tends to be screened by the spatial redistribution of the electrons present in the system, which self-consistently reduces the EPC strength [11-13]. Screening is expected to be much more effective in the adiabatic regime-since, then, electrons are able to equilibrate sufficiently fast in response to the ionic motion - and a much weaker EPC is accordingly expected in this regime as compared to the anti-adiabatic one. Conversely, if the nature of the EPC is described in terms of a vector potential, which, as we mentioned, is the case for some of the phonons in graphene $[14,15]$, screening cannot influence EPC $[7,16]$ (just like no spatial distribution of charge carriers can screen a magnetic field). In this case, EPC is not expected to be significantly affected by the presence of charge carriers.

In semiconducting materials hosting only a small density of mobile charges, electrostatic screening is poor, and a strong electron-phonon interaction is expected. The addition of charge carriers (e.g., by doping) can drastically improve screening and cause the strength of the electronphonon interaction to decrease, as observed in a variety of semiconductors (e.g., $\mathrm{SrTiO}_{3}$ [17-19] and other transition metal oxides [20]). For those cases in which electronphonon interactions cannot be screened, one would expect no effect from the addition of charge carriers, as in graphene [7]. However, there are virtually no cases in which the strength of the electron-phonon interaction in a semiconductor is expected to increase significantly upon adding charge carriers.

In contrast to this established understanding, here we uncover a yet unidentified mechanism that causes a very significant strengthening - and not a weakening — of EPC in atomically thin semiconductors upon increasing electron density. Our work relies on a joint experimental and theoretical investigation of Raman spectroscopy performed on monolayers and bilayers of different semiconducting transition metal dichalcogenides (TMDs; $\mathrm{MoS}_{2}, \mathrm{WS}_{2}$, and $\mathrm{WSe}_{2}$ ) as a function of the density of accumulated charge carriers. By integrating all these atomically thin layers in ionic-liquid-gated field-effect transistors (FETs), we have measured the evolution of the Raman spectrum as a function of electron and hole density up to approximately $5 \times 10^{13} \mathrm{~cm}^{-2}$. In all investigated monolayers and bilayers, the experiments reveal unambiguously that the electronphonon interaction systematically softens out-of-plane vibrational modes only when electrons are accumulated in the FET channel while leaving them unaffected upon hole doping. To identify the origin of this unexpected electron-hole asymmetry, we perform density-functionaltheory (DFT) calculations in a realistic framework that accounts for the reduced dimensionality of the materials and for the presence of a finite density of charge carriers [21]. The calculations allow us to conclude that the observed phonon softening originates from a pronounced increase in the EPC strength that occurs whenever the charge carriers in the systems simultaneously populate two inequivalent valleys. Besides accounting for the observed electronhole asymmetry (as a direct consequence of the different valley structure in the conduction and valence bands of atomically thin semiconducting TMDs), this finding reveals an aspect of EPC that has not yet been appreciated. Specifically, the strengthening of EPC is caused by charge transfer between inequivalent valleys with opposite deformation potentials. Being local in real space, this intervalley charge transfer decreases the spatial variation of charge associated with the phonon perturbation, making it insensitive to screening. The phenomenon-expected to be of rather general validity - has not been identified so far, most likely because the models commonly used to describe electron-phonon interaction theoretically do not consider the presence of multiple inequivalent valleys. Equipped with the understanding resulting from the work presented here, we discuss how existing experiments on superconductivity in TMDs (both gate-induced [22-25] and spontaneously occurring [26-30]) can be viewed as providing evidence correlating the enhancement in electron-phonon interactions due to multivalley populations to the occurrence of the superconducting state.

\section{EXPERIMENTAL RESULTS}

Our experiments consist in measurements of the Raman spectrum of monolayers and bilayers of different semiconducting TMDs ( $\mathrm{WSe}_{2}, \mathrm{WS}_{2}$, and $\mathrm{MoS}_{2}$ ) as a function of carrier density. The density and polarity of charge carriers is varied continuously by employing these atomically thin crystals as active parts of ionic-liquid-gated FETs [see Fig. 1(a) for a schematic illustration]. It has been shown in a multitude of experiments over the last several years that ionic-liquid gating is extremely effective in combination with semiconducting TMDs, as it allows the accumulation of large densities (even in excess of $10^{14} \mathrm{~cm}^{-2}$ ) of both electrons and holes in the same device [22,23,31-39]. As a consequence, ambipolar transport can be observed upon sweeping the gate voltage $V_{G}$ [see Fig. 2(a) for an example]. In the present context, the occurrence of ambipolar transport is useful because it allows us to obtain a fairly accurate estimate of the carrier density in the devices used for the Raman measurements in situ. In practice, we record the Raman spectrum at many different values of applied gate voltage and - as we vary $V_{G}$-we also apply a small bias voltage $\mathrm{V}_{\mathrm{SD}}$ between the source and the drain electrode to measure the current $\mathrm{I}_{\mathrm{SD}}$ passing through the transistor channel. This process allows us to determine the 


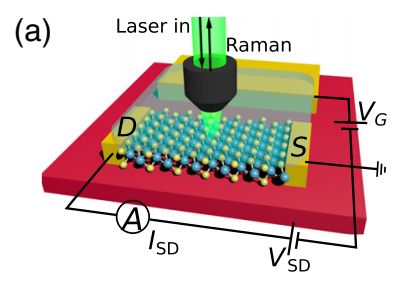

(b)

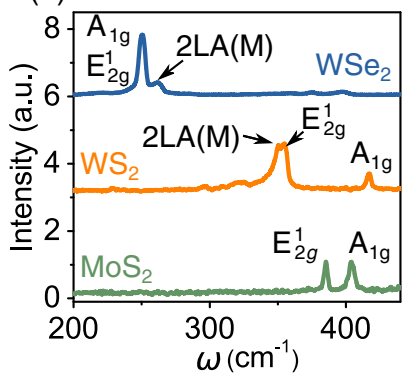

(d)
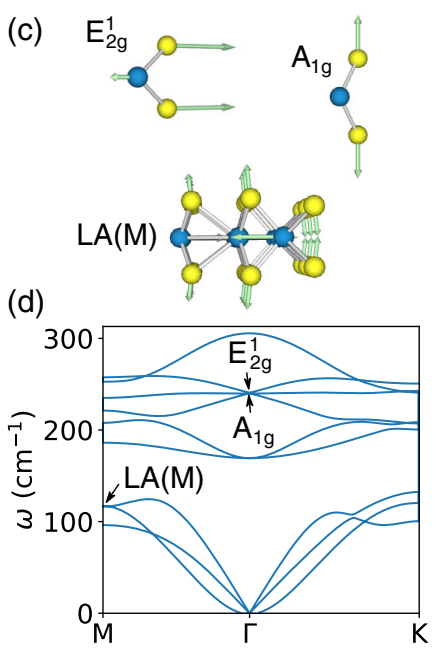

same nomenclature as in the bulk, these are the in-plane $\left(E_{2 g}\right)$ and the out-of-plane $\left(\mathrm{A}_{1 \mathrm{~g}}\right)$ modes, as indicated in Fig. 1(b), which are seen in all group-VI TMDs. The displacement pattern of these phonon modes is schematically reported in Fig. 1(c). For $\mathrm{WS}_{2}$ and $\mathrm{MoS}_{2}$, these features are spectrally separated, while for $\mathrm{WSe}_{2}$, the modes hybridize due to an accidental degeneracy in the phonon dispersion relation and appear as a single peak at about $250 \mathrm{~cm}^{-1}$. The degeneracy is also present in the calculated phonon dispersion relation of $\mathrm{WSe}_{2}$ in Fig. 1(d), on which we point to the modes observed in the Raman spectroscopy data. For most peaks, the observed frequency corresponds to the value of the corresponding mode at the $\Gamma$ point. However, one of the peaks, visible in the spectra of both $\mathrm{WSe}_{2}$ and $\mathrm{WS}_{2}$ and labeled 2LA(M) [see Fig. 1(b)], is due to a double resonant process $[45,46]$, which occurs because the frequency of the laser used in our measurements matches an electronic transition $(\mathrm{C}$ absorption for $\mathrm{WSe}_{2}$ and the $\mathrm{B}$ exciton for $\mathrm{WS}_{2}$ ) [47]. Although it is not the main point of interest here, the possibility to observe this mode is relevant because it allows the investigation of the coupling between electrons and phonons at finite momentum, i.e., away from the center of the Brillouin zone, as indicated by the corresponding label LA(M) in Fig. 1(d).

To illustrate the results of our Raman spectroscopy measurements as a function of accumulated charge density, we start by discussing the case of $\mathrm{WSe}_{2}$ monolayers. The source-drain current $\mathrm{I}_{\mathrm{SD}}$ as a function of $V_{G}$ (for $\mathrm{V}_{\mathrm{SD}}=50 \mathrm{mV}$ ), measured in the device used for the Raman measurements, is presented in Fig. 2(a) and exhibits clear ambipolar behavior. The increase of $\mathrm{I}_{\mathrm{SD}}$ at positive values of $V_{G}$ indicates accumulation of electrons in the FET channel, while the increase of $\mathrm{I}_{\mathrm{SD}}$ at negative $V_{G}$ corresponds to the accumulation of holes. As explained above, we use these measurements to estimate the density of electrons and holes as a function of $V_{G}$. Figure 2(b) shows the Raman spectra measured for selected values of $V_{G}$ indicated by the vertical dashed lines in Fig. 2(a). For positive values of $V_{G}$, electrons are accumulated (green and red lines, i.e., $V_{G}=+1.2$ and $+1.5 \mathrm{~V}$, corresponding, respectively, to $n_{e} \sim 1.5 \times 10^{13}$ and $\left.2.5 \times 10^{13} \mathrm{~cm}^{-2}\right)$; for negative $V_{G}$ values, the gate accumulates holes (orange line, i.e., $V_{G}=-1.5 \mathrm{~V}, \quad$ corresponding to $n_{h} \sim$ $6 \times 10^{13} \mathrm{~cm}^{-2}$ ); or $V_{G}=0 \mathrm{~V}$ (blue line), the chemical potential is in the gap, and the semiconductor is neutral [48]. Upon electron accumulation, the Raman peak originating from the hybridized $\mathrm{A}_{1 \mathrm{~g}} / \mathrm{E}_{2 \mathrm{~g}}$ mode exhibits a clear softening, shifting towards lower wave numbers by an amount $\Delta \omega \simeq 3-4 \mathrm{~cm}^{-1}$, as well as a decrease in intensity with respect to $V_{G}=0 \mathrm{~V}$. In contrast, accumulation of holes leaves the Raman spectrum virtually unchanged: The peak does not exhibit any appreciable shift, and only its height decreases slightly. The same trends upon varying $V_{G}$ are observed for the resonant Raman peak originating from the 2LA(M) mode, shown in the right panel of Fig. 2(b) [49]. 

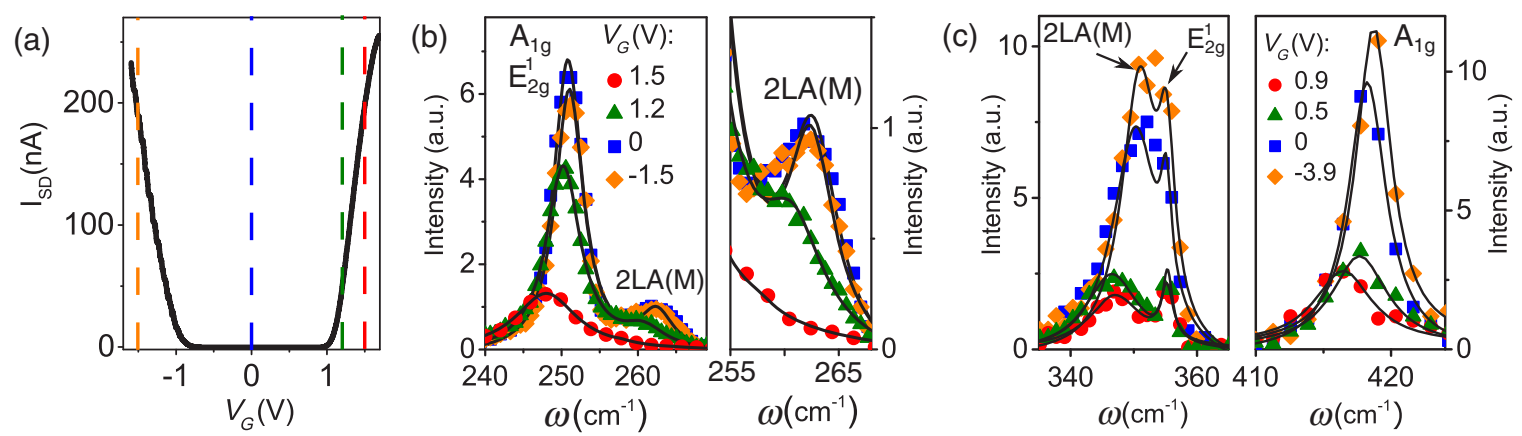

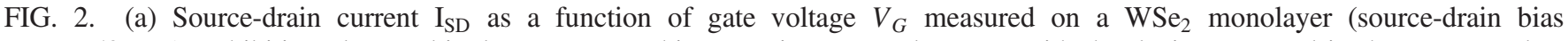
$\mathrm{V}_{\mathrm{SD}}=50 \mathrm{mV}$ ), exhibiting clear ambipolar transport. This curve is measured in situ, with the device mounted in the setup used to perform Raman spectroscopy, and it enables us to estimate the density of carriers at any given value of $V_{G}$ as discussed in the main text. (b) Left panel: Raman spectrum of the $\mathrm{WSe}_{2}$ monolayer at selected gate voltages indicated in the legend and corresponding to the values of $V_{G}$ marked with the dashed lines of different colors in panel (a). The peak near $250 \mathrm{~cm}^{-1}$ originates from the degenerate $\mathrm{A}_{1 \mathrm{~g}}$ and $\mathrm{E}_{2 \mathrm{~g}}$ modes, and the one near $250 \mathrm{~cm}^{-1}$ is due to a resonant electronic process involving the LA(M) mode. In all cases, the black lines are fits of the data to Lorentzian-shaped peaks centered around the energy of the different modes identified. (b) Right panel: Zoom-in on the spectral region around the peak due to the resonant process involving the LA(M) mode. (c) Left panel: Peaks in the Raman spectrum of the $\mathrm{WS}_{2}$ bilayer originating from the $\mathrm{E}_{2 \mathrm{~g}}$ and $2 \mathrm{LA}(\mathrm{M})$ modes for different values of gate voltage (see the legend in the right panel). (c) Right panel: Peak in the Raman spectrum due to the $\mathrm{A}_{1 \mathrm{~g}}$ mode measured at the gate voltages indicated in the legend (black lines are fits of the data obtained by introducing a Lorentzian-shaped peak for each one of the modes involved). As for the WSe $\mathrm{m}_{2} \mathrm{monolayer}$, the spectra are strongly affected upon electron accumulation; the $\mathrm{A}_{1 \mathrm{~g}}$ and $2 \mathrm{LA}(\mathrm{M})$ modes shift to lower wave numbers (i.e., softening) and decrease in intensity, whereas they are left virtually unchanged upon hole accumulation. The position of the $\mathrm{E}_{2 \mathrm{~g}}$ peak remains constant for both electron and hole accumulation.

Since in $\mathrm{WSe}_{2}$ monolayers the degeneracy of the $\mathrm{E}_{2 \mathrm{~g}}$ and $\mathrm{A}_{1 \mathrm{~g}}$ modes prevents us from studying separately the evolution of the two individual Raman peaks upon accumulation of charge carriers, in Fig. 2(c), we show data for bilayer $\mathrm{WS}_{2}$, in which these modes are well separated in frequency. The corresponding Raman peak positions at $V_{G}=0 \mathrm{~V}$ are located, respectively, at $\omega=355 \mathrm{~cm}^{-1}$ [Fig. 2(c), left panel] and $417 \mathrm{~cm}^{-1}$ [Fig. 2(c), right panel]. The same figures also show the Raman spectra measured at selected values of $V_{G}$, in a range of carrier density somewhat larger than for monolayer $\mathrm{WSe}_{2}$. For values of $V_{G}$ corresponding to hole accumulation, only a negligible shift in position and, at most, a small change in amplitude are seen for all peaks. In contrast, upon electron accumulation, the $\mathrm{A}_{1 \mathrm{~g}}$ and the $2 \mathrm{LA}(\mathrm{M})$ modes exhibit a clear softening, similarly to what is observed in $\mathrm{WSe}_{2}$ (the position of the $E_{2 g}$ mode remains unaffected even for electron accumulation).

Figure 2 reveals a number of clear trends of the Raman active modes in monolayer $\mathrm{WSe}_{2}$ and bilayer $\mathrm{WS}_{2}$, such as the insensitivity of all phonon modes to hole accumulation and the softening of out-of-plane modes upon electron accumulation. We have performed gate-dependent Raman spectroscopy measurements on monolayers and bilayers of $\mathrm{WSe}_{2}, \mathrm{WS}_{2}$, and $\mathrm{MoS}_{2}$ following a procedure identical to the one described above, and found that these trends are generically present in all the investigated atomically thin crystals. To quantitatively illustrate and compare the results of measurements performed on different systems, we plot for each one of them the change $\Delta \omega$ in the frequency of the different phonon modes relative to the value measured at threshold for electron or hole accumulation (i.e., for each system, we plot $\Delta \omega$ versus $V_{G}-V_{\mathrm{th}}^{\mathrm{e}}$ and $V_{G}-V_{\mathrm{th}}^{\mathrm{h}}$ ). The results of these measurements for the $\mathrm{A}_{1 \mathrm{~g}}$ mode in all the investigated monolayers and bilayers are summarized in Figs. 3(a) and 3(b). The data for the $E_{2 g}$ mode in monolayers are shown in Fig. 3(c). It is clear from this systematic experimental analysis-which goes well beyond what had been done before now [50,51] - that the same behavior is common to all atomically thin group-VI TMDs.

To quantify the strength of the influence of electron and hole accumulation on the frequency of the different phonons, we plot, for all systems investigated, the frequency shift of the $A_{1 \mathrm{~g}}$ and $\mathrm{E}_{2 \mathrm{~g}}$ modes measured at the highest value of gate voltage applied (relative to the corresponding threshold voltage), $\left|V_{G}-V_{\mathrm{th}}^{\mathrm{e}, \mathrm{h}}\right|=0.6 \mathrm{~V}$, for which we have obtained data for both monolayers and bilayers, as well as for electron and hole accumulation. Figure 3(d) demonstrates clearly that, for both monolayers and bilayers, only Raman active phonon modes with an out-of-plane component of the atomic displacement are influenced by the presence of charge carriers, whereas the $E_{2 g}$ mode - for which the atomic displacement is in plane-is left unaffected by the presence of free charge. The data also make the strong electron-hole asymmetry in the softening of the out-of-plane modes very apparent for all the different TMDs investigated. The order of magnitude of the effect-which is what we discuss here-is the same 

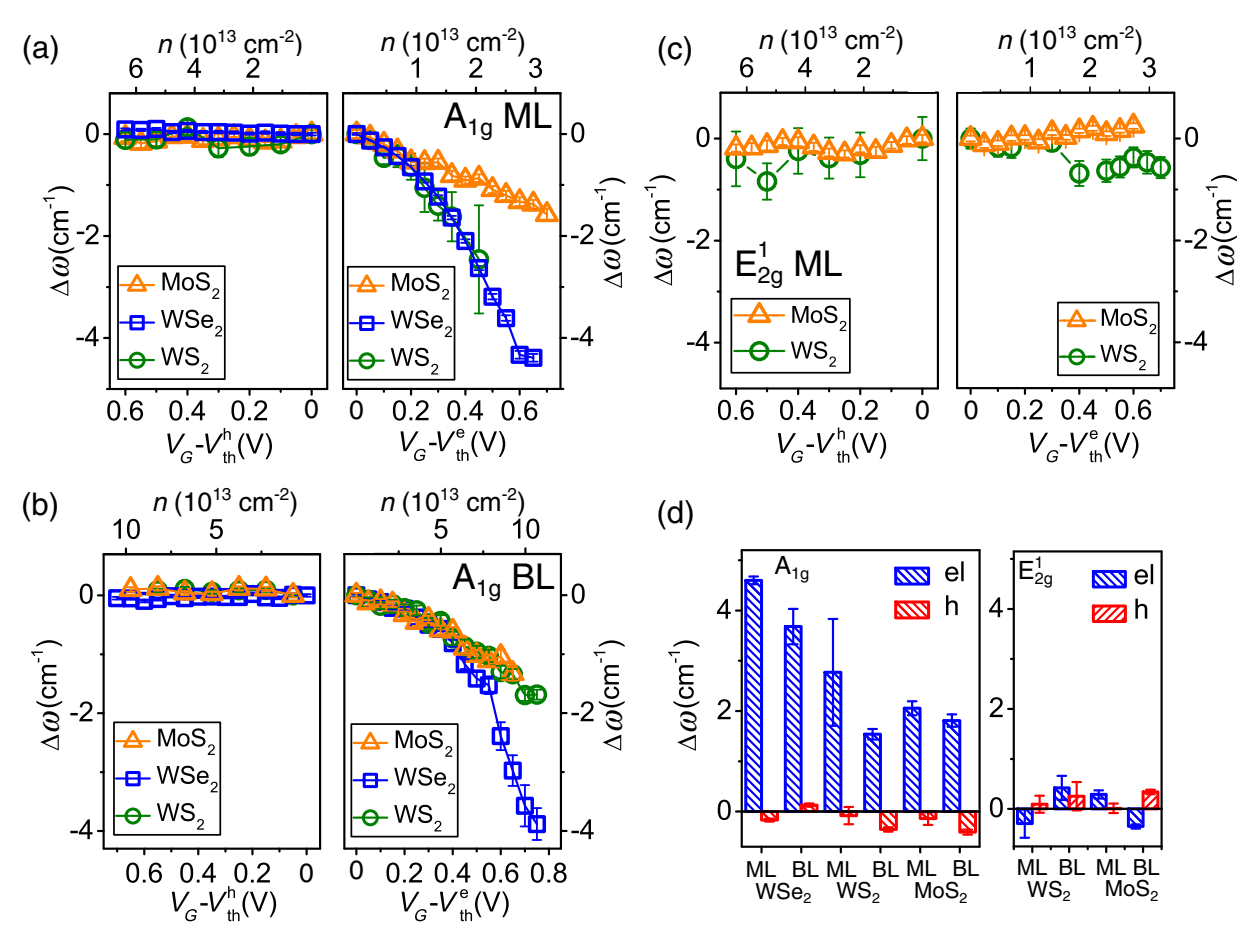

FIG. 3. (a) Shift $\Delta \omega$ of the $\mathrm{A}_{1 \mathrm{~g}}$ peak position in $\mathrm{MoS}_{2}$ (orange open triangles), WSe $\mathrm{C}_{2}$ (blue open squares), and WS (green open circles) monolayers as a function of gate voltage (bottom axis), relative to the threshold voltage for hole accumulation (left panel) and for electron accumulation (right panel). The values of electron and hole density corresponding to the applied gate voltage-estimated using the capacitance of the ionic liquid gate, $n_{e, h}=(1 / e) C\left|V_{G}-V_{\mathrm{th}}^{\mathrm{e}, \mathrm{h}}\right|$-is indicated on the top axis (the capacitance values are largely determined by the density of states, as discussed in Ref. [48]). The position of the Raman peak originating from the $\mathrm{A}_{1 \mathrm{~g}}$ mode remains unchanged for all TMD monolayers upon hole accumulation and shifts upon electron accumulation. (b) Same as panel (a) for the $A_{1 g}$ mode of bilayers of the same TMD compound, $\mathrm{MoS}_{2}$ (orange open triangles), $\mathrm{WSe}_{2}$ (blue open squares), and $\mathrm{WS}_{2}$ (green open circles). The trends and the order of magnitude of the frequency softening are identical in monolayers and bilayers. (c) The $\mathrm{E}_{2 \mathrm{~g}}$ peak in monolayer $\mathrm{MoS}_{2}$ (orange open triangles) and $\mathrm{WS}_{2}$ (green open circles) remains constant irrespective of the applied gate voltage (the left panel corresponds to hole accumulation and the right one to electron accumulation; for $\mathrm{WSe}_{2}$, the investigation of the $\mathrm{E}_{2 \mathrm{~g}}$ mode is prevented by the degeneracy with the $\mathrm{A}_{1 \mathrm{~g}}$ mode). The same insensitivity of the $\mathrm{E}_{2 \mathrm{~g}}$ mode to charge accumulation is seen in bilayers (not shown). (d) To summarize the effect of electron and hole accumulation on the softening of the $\mathrm{A}_{1 \mathrm{~g}}$ and $\mathrm{E}_{2 \mathrm{~g}}$ phonon modes, we compare the absolute shift of the peak position at the highest gate voltage value (relative to the corresponding threshold voltage) for all different investigated monolayer and bilayer TMDs, $\left|V_{G^{-}} \mathrm{V}_{\mathrm{th}}^{\mathrm{e} h \mathrm{~h}}\right|=0.6 \mathrm{~V}$. The left and right panels summarize the values measured for the $\mathrm{A}_{1 \mathrm{~g}}$ and $\mathrm{E}_{2 \mathrm{~g}}$ modes, respectively, for either electrons (blue bars) or holes (red bars). It is clear from these plots that only the $\mathrm{A}_{1 \mathrm{~g}}$ modes (with an out-of-plane motion component of the atomic displacement) are affected by introduction of carriers and only if electrons are accumulated.

in all cases, corresponding to a shift of $2-4 \mathrm{~cm}^{-1}$ for the $\mathrm{A}_{\mathrm{lg}}$ mode (for a more detailed quantitative analysis, it should be recalled that-since the value of the capacitance used is different for monolayers and bilayers and for electron and hole accumulation-the same value of gate voltage relative to threshold does not correspond to the same carrier density [48]).

\section{THEORETICAL ANALYSIS}

The experiments discussed in the previous section reveal that a strong electron-hole asymmetry in the softening of the phonon frequency is a generic property of atomically thin group-VI semiconducting TMDs. Here, we present a theoretical analysis showing that the strength of the electron-phonon interaction in these materials is governed by whether or not multiple valleys (the $\Gamma$ and $\mathrm{K}$ valleys in the valence band and the $\mathrm{K}$ and $\mathrm{Q}$ valleys in the conduction band; see Fig. 4) are simultaneously populated by the accumulated charge carriers. It follows from this analysis that it is the rather different valley structure of the conduction and the valence bands that is ultimately responsible for the difference in the coupling to phonons of electrons and holes, and this difference causes the observed electron-hole asymmetry in the softening of the phonon frequencies.

Our conclusions are based on DFT and densityfunctional perturbation theory (DFPT) calculations, which provide an extremely powerful tool to identify all trends in the observed dependence of the phonon frequency on the density of accumulated charge. DFT, however, cannot reproduce the experiments at a detailed quantitative level 


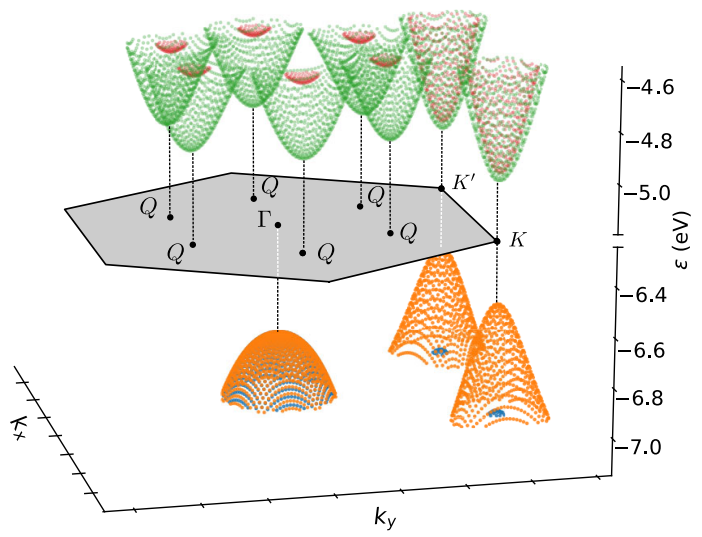

FIG. 4. The low-energy valley structure of the conduction and valence bands is common to all the TMD monolayers investigated here. This figure illustrates it by showing the low-energy parts of the conduction and valence bands of $\mathrm{WS}_{2}$ monolayers (the grey shaded hexagon represents the Brillouin zone). The top of the valence band (shown in orange and blue) is formed by a single spin-degenerate valley around $\Gamma$ and a spin-split, twofold degenerate valley at the $\mathrm{K} / \mathrm{K}^{\prime}$ point. The bottom of the conduction bands (represented in green and red) is composed of an almost spin-degenerate valley at the $\mathrm{K} / \mathrm{K}^{\prime}$ point (with degeneracy two) and a sixfold degenerate spin-split valley approximately midway between $\Gamma$ and $\mathrm{K}$, normally referred to as the $\mathrm{Q}$ point. Note that time-reversal-related valleys $\left(\mathrm{K} / \mathrm{K}^{\prime}\right.$ or $\left.\mathrm{Q} / \mathrm{-Q}\right)$ have opposite out-of-plane spin polarization.

because a full quantitative agreement would require a precise prediction of the relative energetic alignment of the valleys present in the valence and conduction band, well known to be extremely sensitive to multiple parameters [52-56], from the choice of exchange-correlation functional to details of the crystal structure and screening. Indeed, even experimental attempts to precisely determine the energetics of the different valleys seem to give scattered results, dependingfor instance - on the substrate employed in the experiments (see Table I). The situation is even more complex for bilayers, where the electric field associated with the FET doping influences the relative energy of the valleys in a way that cannot be precisely predicted at this stage. As such, our analysis aims exclusively at exploiting the possibility to tune parameters and conditions in the controlled environment of DFT simulations to identify the physical processes that explain our experimental results, and not at reproducing, in quantitative detail, the observed carrier density dependence of the phonon frequencies. In view of the subtle effects caused by the gate electric field in bilayers, we focus our theoretical analysis exclusively on TMD monolayers.

We first note that the softening is expected when describing electron-phonon interactions within the BornOppenheimer approximation, where electrons remain, at all times, in the ground state corresponding to the instantaneous lattice configuration. This case implies that phonons can be considered as static perturbations acting on the electrons, which in turn affect the vibrational properties of the system. In this regime, the expression for the change in frequency of a phonon at $\Gamma$ with respect to the neutral semiconducting case reads (see Appendix B and Refs. [68-70]):

$$
\Delta \omega_{\nu} \approx-N\left(\varepsilon_{F}\right)\left\langle g_{\nu}^{2}\right\rangle_{\mathrm{FS}}
$$

Here, we assume the limit of zero temperature, $N\left(\varepsilon_{F}\right)$ is the density of states at the Fermi energy $\varepsilon_{F}$, and $\left\langle g_{\nu}^{2}\right\rangle_{\mathrm{FS}}$ is the screened EPC (squared) for the $\nu$ th phonon mode averaged over the Fermi surface [see Eq. (B9) in Appendix B]. It follows that softening $\left(\Delta \omega_{\nu}<0\right)$ is expected only for phonon modes that are strongly coupled with the electronic states on the Fermi surface. For instance, this is not the case of the pure $E_{2 g}$ longitudinal optical mode, which affects electrons by generating a long-range scalar potential (known as the Fröhlich interaction) that, in the presence of free carriers (either electrons or holes), is perfectly screened in the long-wavelength limit, so that $\left\langle g_{\nu}^{2}\right\rangle_{\mathrm{FS}} \approx 0$.

The Born-Oppenheimer approximation is a good description only for systems that are in the adiabatic limit.

TABLE I. Energy separation between the top of the valence band at $\mathrm{K}$ and $\Gamma\left(\Delta E_{\Gamma \mathrm{K}}\right)$ and between the bottom of the conduction bands at $\mathrm{K}$ and $\mathrm{Q}\left(\Delta E_{\mathrm{KQ}}\right)$ in the monolayer TMDs investigated in this work. Experimental results are taken from angle-resolved photoemission spectroscopy (ARPES) measurements in the literature. DFT data refer to calculations performed with the same exchange-correlation functional (PBE [57]) on the DFT-relaxed crystal structure.

\begin{tabular}{lccc}
\hline \hline & \multicolumn{3}{c}{$\Delta E_{\Gamma \mathrm{K}}(\mathrm{meV})$} \\
\cline { 2 - 4 } & $\mathrm{MoS}_{2}$ & $\mathrm{WS}_{2}$ & $\mathrm{WSe}_{2}$ \\
\hline Experiment & $150[58], 310[55,59], 140[60]$ & $510[61], 240[62], 300[63], 390[60]$ & $560[64], 500[65], 890[53], 620[60]$ \\
DFT & $55^{\mathrm{a}}, 46[54], 50[66]$ & $230^{\mathrm{a}}, 270[54], 220[66]$ & $490^{\mathrm{a}}, 506[54], 530[43,66]$ \\
\hline & & $\Delta E_{\mathrm{KQ}}(\mathrm{meV})$ & \\
\cline { 2 - 4 } & $\mathrm{MoS}_{2}$ & $\mathrm{WS}_{2}$ & $\mathrm{WSe}_{2}$ \\
\hline Experiment & $\ldots$ & $\ldots$ & $<30[60]$ \\
DFT & $240^{\mathrm{a}}, 246[54]$ & $100^{\mathrm{a}}, 58[54]$ & $40^{\mathrm{a}}, 32[54], 50[43]$ \\
\hline \hline
\end{tabular}

${ }^{\mathrm{a}}$ This work and Ref. [67]. 
It requires the phonon energy $\hbar \omega_{\nu}$ to be much smaller than the carrier relaxation rate $\hbar / \tau\left(\omega_{\nu} \tau \ll 1\right)$, where $\tau$ is the carrier momentum-relaxation lifetime. The value of $\tau$ can be estimated from the experimentally determined carrier mobility that — under the conditions of the experiments-is typically $\mu \approx 15 \mathrm{~cm}^{2} / \mathrm{Vs}$ for both electrons and holes. When compared to the characteristic phonon frequencies probed in our experiments, the resulting value of $\tau \approx 10 \mathrm{fs}$ gives $\omega_{\nu} \tau \leq 1$. The Born-Oppenheimer approximation is therefore fairly well justified. Note also that, even when $\omega_{\nu} \tau \simeq 1$, theory allows the phonon softening to be estimated as $[10,69,71]$

$$
\Delta \omega_{\nu}^{(\tau)} \approx \frac{\Delta \omega_{\nu}}{1+\left(\omega_{\nu} \tau\right)^{2}}
$$

where $\Delta \omega_{\nu}$ is given by Eq. (1). It follows directly from this expression that the adiabatic limit represents an upper bound on the prediction for the phonon softening, possibly overestimating the effect measured in experiments by at most a factor of 2 .

Since DFT calculations of phonon frequencies are performed within the Born-Oppenheimer approximation, they are an appropriate tool to simulate the softening in monolayer TMDs. We first investigate which phonon modes are sensitive to accumulation of charge carriers by computing phonon dispersions of neutral, electrondoped and hole-doped monolayer TMDs using DFPT [72]. Since simulations require overall charge neutrality, the accumulated charge is compensated by the presence of gate electrodes, and the correct boundary conditions for 2D materials are provided by cutting off the Coulomb interactions in the direction perpendicular to the layers [21]. In these initial calculations, spin-orbit coupling is not included, and a relatively large electronic temperature is used to smear the Fermi surface (see Appendix A).

Representative DFPT results are shown in Fig. 5 for $\mathrm{WS}_{2}$. When a finite density of carriers is included, we observe a softening in various branches at several phonon wave vectors. In particular, we see that the $A_{1 g}$ mode softens at $\Gamma$ both for electron and hole accumulation, and at $\mathrm{M}$ for electron accumulation only. Similarly, the frequencies of the in-plane acoustic modes (LA and TA) decrease at $\mathrm{K}$ upon both electron and hole accumulation (indicating a strong coupling consistent with Ref. [73]) and at M exclusively for electron accumulation. On the contrary, the $E_{2 g}$ mode remains unaffected. For all the phonon modes detected in the experiments, therefore, the simulations succeed in reproducing which modes soften upon accumulation of charge carriers. However, the overall agreement between calculations and experiments is poor. The magnitude of the softening is largely overestimated, well beyond the factor $\left[1+\left(\omega_{\nu} \tau\right)^{2}\right]$ accounting for deviations from the purely adiabatic approximation of DFT.

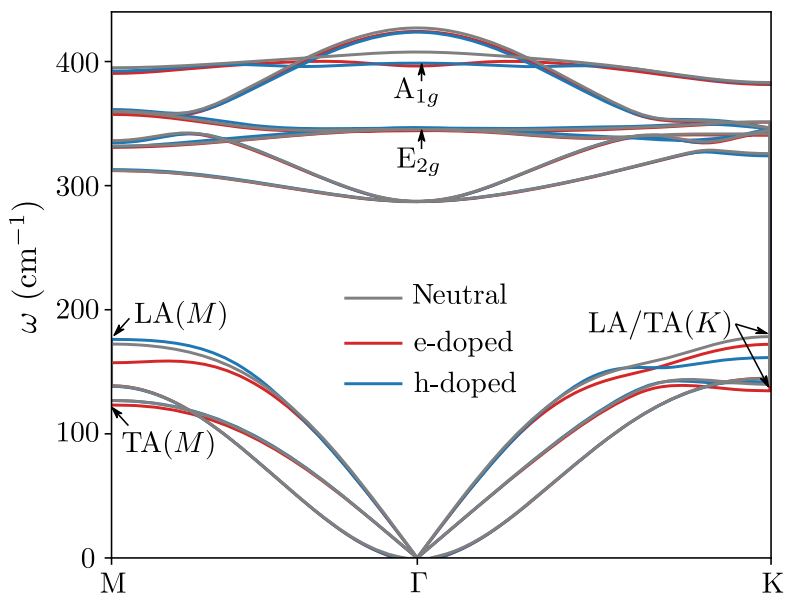

FIG. 5. Dispersion relations of the different phonons branches, calculated as described in the main text, along the high-symmetry path $\mathrm{M}-\Gamma-\mathrm{K}$ of monolayer $\mathrm{WS}_{2}$. Lines of different colours correspond to either neutral (grey lines) monolayers, or monolayers under electron (blue lines), and hole (red lines) accumulation (calculations are done for 0.02 electrons or holes per unit cell).

In addition, softening appears also upon hole doping for the $\mathrm{A}_{1 g}$ mode at $\Gamma$, in contrast to the experimental results.

To investigate the origin of the discrepancy between measurements and simulations, we focus our attention on the $A_{1 g}$ mode [49]. We compute the $A_{1 g}$ frequency at $\Gamma$ as a function of carrier density using a finite-difference DFT scheme (see Appendix A) for the three monolayer TMDs investigated here. At this stage, we make calculations more realistic by including spin-orbit coupling through fully relativistic pseudopotentials and by describing the occupation of electronic states according to the room-temperature Fermi-Dirac distribution (for which we make a much denser sampling of the Brillouin zone). This finer framework is necessary to simulate the valley occupations accurately.

Results are shown in Fig. 6, both for electron and hole accumulation, in a range of carrier densities comparable to that explored in the experiments. In contrast to the very systematic results of the Raman measurements (see Fig. 3), the evolution of the calculated phonon frequency upon electron and hole accumulation is different for the individual TMDs and appears, at first sight, to offer no systematics. In particular, we find that for $\mathrm{WS}_{2}$ [Fig. 6(a)], a large softening occurs on the electron side, but the $\mathrm{A}_{1 \mathrm{~g}}$ mode frequency decreases also upon hole doping (see also Fig. 5). For $\mathrm{WSe}_{2}$ [Fig. 6(b)], instead a significant softening is present only for electrons and not for holes, whereas the opposite is true for $\mathrm{MoS}_{2}$ [Fig. 6(c)]. Despite this seemingly erratic behavior, there is a clear and systematic correlation between the softening and the fractional occupation $P$ of the different valleys plotted in the bottom panels of Fig. 6 .

In all cases, a strong softening of the $A_{1 g}$ mode occurs only when two inequivalent valleys are simultaneously 

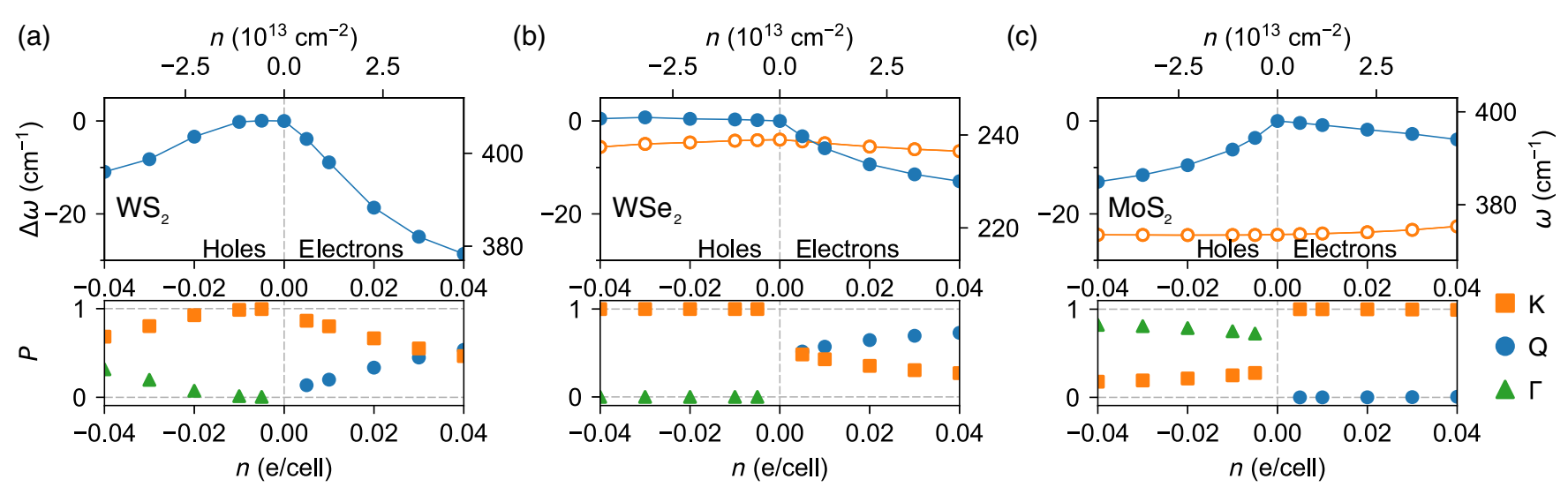

FIG. 6. Top panels: Results of ab initio calculations showing the dependence of the $\mathrm{A}_{1 \mathrm{~g}}$ mode frequency (blue circles) on carrier density $n$ in monolayer $\mathrm{WS}_{2}$ (a), $\mathrm{WSe}_{2}$ (b), and $\mathrm{MoS}_{2}$ (c), for either electron $(n>0)$ or hole $(n<0)$ accumulation, as indicated in each panel. The range of carrier density considered in the calculations roughly corresponds to that explored in the experiments [see Fig. 2(a)]. For $\mathrm{WSe}_{2}$ and $\mathrm{MoS}_{2}$, we also plot the results for the $\mathrm{E}_{2 \mathrm{~g}}$ mode (orange empty circles), which is found not to shift, in agreement with experiments (for $\mathrm{WS}_{2}$, the $\mathrm{E}_{2 \mathrm{~g}}$ mode is degenerate with the $\mathrm{A}_{1 \mathrm{~g}}$ mode, which experimentally prevents its separate determination upon varying carrier density). Lower panels: Relative fractional occupation $P$ of the different valleys upon hole and electron accumulation, as extracted from $a b$ initio calculations done to obtain the density dependence of the phonon frequencies shown in the corresponding top panels. Different symbols refer to the K (orange squares), Q (blue circles), and $\Gamma$ (green triangles) valleys. Note how in all cases-i.e., for all the different monolayers and upon both electron and hole accumulation-there is a perfect correlation between the shift of the $\mathrm{A}_{1 \mathrm{~g}}$ mode and the multiple occupation of valleys (i.e., the $\mathrm{K}$ and $\Gamma$ valleys in the valence band upon hole accumulation, and the $\mathrm{K}$ and $\mathrm{Q}$ valleys in the conduction band upon electron accumulation).

occupied, whereas the frequency of this mode remains constant whenever the accumulated carriers exclusively occupy one of the valleys. Specifically, the rapid decrease of the $\mathrm{A}_{1 \mathrm{~g}}$ frequency upon electron doping in $\mathrm{WS}_{2}$ and $\mathrm{WSe}_{2}$ originates from the simultaneous occupation of the $\mathrm{K}$ and $\mathrm{Q}$ valleys [see Figs. 6(a) and 6(b), respectively]. Consistently with this idea, in $\mathrm{MoS}_{2}$, only the $\mathrm{K}$ valley is populated, and no significant softening is observed. Analogous trends are seen upon hole accumulation: A large softening is present in $\mathrm{MoS}_{2}$, for which the $\mathrm{K}$ and $\Gamma$ valleys are simultaneously occupied, whereas no softening is visible in $\mathrm{WSe}_{2}$ where holes populate only the $\mathrm{K}$ valley [see Figs. 6(c) and 6(b), respectively]. The case of $\mathrm{WS}_{2}$ monolayers [Fig. 6(a)] confirms this notion. In this case, the frequency of the $A_{1 g}$ mode is insensitive to hole doping for small concentrations - when only the $\mathrm{K}$ valley is occupied-and starts to soften past 0.02 holes/unit cell, when holes begin to populate both the $\mathrm{K}$ and the $\Gamma$ valley. In all cases, a very pronounced softening is always seen when two distinct valleys are populated, whereas whenever only one valley is populated, the change in phonon frequency is typically at least one order of magnitude smaller.

It follows from these considerations that the energy separation between valleys, which governs their relative occupation, plays an essential role in determining the magnitude of the shift in phonon frequencies (i.e., the EPC strength) upon carrier accumulation. To confirm this conclusion-and to provide further support for the effect of multivalley populations - we have performed additional calculations exploiting the extreme sensitivity of TMD band structures to the in-plane lattice parameter and the vertical positions of the chalcogen atoms [55]. The goal of these calculations is to artificially tune the energy separation between valleys in order to check whether, as we expect, the softening of the $\mathrm{A}_{1 \mathrm{~g}}$ mode is affected significantly. We analyze the behavior of $\mathrm{MoS}_{2}$ monolayers using the experimentally reported crystal structure (rather than the fully relaxed one obtained by minimizing forces and stresses at the DFT level), for which the energy separation $\Delta E_{\Gamma \mathrm{K}}$ between the $\mathrm{K}$ and $\Gamma$ valleys in the valence band increases from $55 \mathrm{meV}$ to $220 \mathrm{meV}$ [see Fig. 7(c)], and the energy difference $\Delta E_{\mathrm{KQ}}$ between the $\mathrm{K}$ and $\mathrm{Q}$ valleys in the conduction band decreases from $240 \mathrm{meV}$ to $30 \mathrm{meV}$. To reduce the spurious effects of thermal broadening in these calculations, we intentionally determine band occupations by using a cold-smeared distribution [74] with $T=150 \mathrm{~K}$, instead of a broad Fermi-Dirac distribution at $T=300 \mathrm{~K}$ as above.

The outcome of the calculations is illustrated in Fig. 7, where we compare the carrier-density dependence of (a) the frequency of the $A_{1 \mathrm{~g}}$ mode and (b) the fractional valley occupations obtained with either the DFT-relaxed structure [empty symbols, see also Fig. 6(c)] or the experimentally reported one (full symbols). It is apparent that the results are distinctly different in the two cases. Upon hole accumulation, a large frequency shift and a simultaneous population of the $\mathrm{K}$ and $\Gamma$ valleys are present for calculations performed using the DFT-relaxed structure. In contrast, no shift in frequency occurs in the calculations performed with the experimentally reported crystal 
(a)

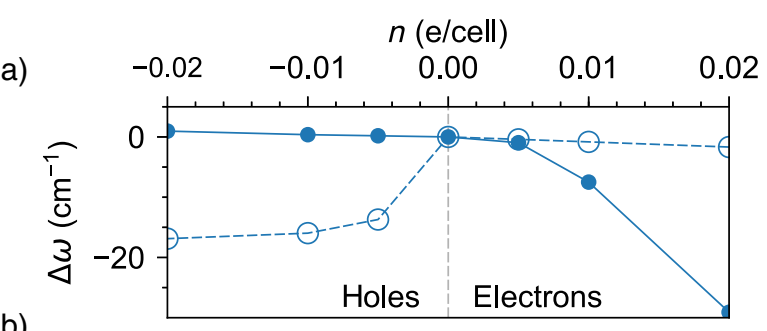

(b)

Q

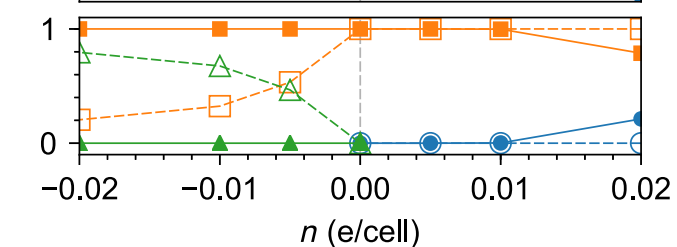

(c)

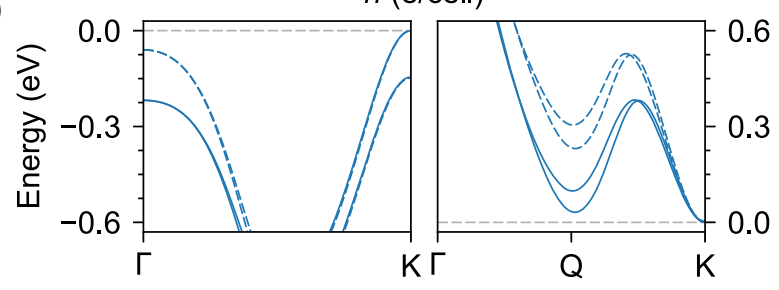

FIG. 7. Effect of the crystal structure of monolayer $\mathrm{MoS}_{2}$ on the softening of the $\mathrm{A}_{1 \mathrm{~g}}$ mode (a) and on the fractional occupation $P$ of the $\mathrm{K}$ (squares), Q (circles), and $\Gamma$ (triangles) valleys (b) upon carrier accumulation. Empty symbols represent quantities obtained by means of $a b$ initio calculations performed using the fully relaxed DFT structure of monolayer $\mathrm{MoS}_{2}$, whereas full symbols represent the same quantities obtained using the experimentally reported structure. Panel (c): Band structure of undoped $\mathrm{MoS}_{2}$ close to the top of the valence bands (left) or to the bottom of the conduction bands (right) calculated using the experimentally reported (continuous lines) or the DFT-relaxed (dashed lines) crystal structure. Note how the different structures very significantly change the distance in energy between the top of the $\mathrm{K}$ and $\Gamma$ valleys in the valence band and between the bottom of the $\mathrm{K}$ and $\mathrm{Q}$ valleys in the conduction band. As discussed in the main text, this distance in energy determines whether or not multiple valleys are occupied upon electron or hole accumulation in the calculations, with a corresponding strong effect on the shift of the $\mathrm{A}_{1 \mathrm{~g}}$ phonon frequency.

structure, where the fractional occupation of the $\mathrm{K}$ valley remains equal to 1 , i.e., only the $\mathrm{K}$ valley is populated. This behavior is exactly what we anticipated as a result of the increased $\Delta E_{\Gamma \mathrm{K}}$ for the experimentally reported crystal structure. Consistently, the reduction in $\Delta E_{\mathrm{KQ}}$ [see Fig. 7(c)] in the experimentally reported structure leads to a non-negligible occupation of the $\mathrm{Q}$ valley in addition to the lowest-lying $\mathrm{K}$ valley, causing a very large enhancement of the $\mathrm{A}_{1 \mathrm{~g}}$ softening upon electron accumulation.

We therefore conclude that the scenario hypothesized above is correct: For all monolayers investigated here, and for both electron and hole accumulation, a very sizable softening of the $\mathrm{A}_{1 \mathrm{~g}}$ mode occurs only when charge carriers populate multiple inequivalent valleys. Through Eq. (1), this result directly implies that the EPC strength for the $\mathrm{A}_{1 \mathrm{~g}}$ mode in semiconducting TMDs is also large only when two valleys are occupied. Such a mechanism has not been identified previously. It is nevertheless extremely likely that this mechanism is at play in many other material systems besides semiconducting TMDs, and it is relevant to explain a variety of physical phenomena (see, e.g., the discussion of superconductivity in Sec. IV).

The insight gained on the importance of the simultaneous occupation of multiple valleys can now be exploited to discuss in more detail the results of our Raman spectroscopy experiments (see Fig. 3). To start with, the complete absence of phonon softening upon hole accumulation indicates that $\Delta E_{\Gamma \mathrm{K}}$ is sufficiently large in TMD monolayers to avoid the partial occupation of the $\Gamma$ valley (in addition to the dominant $\mathrm{K}$ valley), for hole concentrations as large as about $5 \times 10^{13} \mathrm{~cm}^{-2}$. According to the same logic, the phonon softening observed upon electron accumulation indicates that $\Delta E_{\mathrm{KQ}}$ in the conduction band is sufficiently small, so-at room temperature-both the $\mathrm{K}$ and $\mathrm{Q}$ valleys are populated in monolayers of all TMDs upon accumulation of about $10^{-2}$ electrons per unit cell. Additionally, it is clear from Fig. 3(a) that phonon softening upon electron accumulation is much more pronounced in W-based TMD monolayers, indicating that $\Delta E_{\mathrm{KQ}}$ is smaller for $\mathrm{WS}_{2}$ and $\mathrm{WSe}_{2}$ than for $\mathrm{MoS}_{2}$.

All these conclusions are fully consistent with what is known about monolayers of semiconducting TMDs. The energy difference $\Delta E_{\Gamma \mathrm{K}}$ reported in Table I from ARPES measurements is consistent with having only the $\mathrm{K}$ valley occupied upon accumulation of a density of holes reaching up to about $5 \times 10^{13} \mathrm{~cm}^{-2}[64,75,76]$. Similarly, although no sufficiently systematic ARPES study of the conduction band of monolayer TMDs has been reported yet, analogous measurements on the doped surface of the bulk [77] (where doping should be limited to the first few layers) or monolayer [60] $\mathrm{WSe}_{2}$ have shown that it is relatively easy to populate both the $\mathrm{K}$ and $\mathrm{Q}$ valleys in the conduction band (i.e., $\Delta E_{\mathrm{KQ}}$ in monolayer $\mathrm{WSe}_{2}$ is significantly smaller than $\Delta E_{\Gamma \mathrm{K}}$; see Table I). Moreover, the observation of a larger softening in W-based monolayers, associated with the $\mathrm{K}$ and $\mathrm{Q}$ valleys being closer in $\mathrm{WS}_{2}$ and $\mathrm{WSe}_{2}$ than in $\mathrm{MoS}_{2}$, is compatible with the fact that $\Delta E_{\mathrm{KQ}}$ is largely controlled by spin-orbit coupling (which is stronger in W-based compounds).

These considerations also make it clear why the strong sensitivity of the phonon frequencies to the precise energy separation between valleys severely affects the ability of DFT to make reliable quantitative predictions. In particular, the spurious softening predicted on the hole side of $\mathrm{MoS}_{2}$ [see Fig. 6(c)] originates from the large underestimation of $\Delta E_{\Gamma \mathrm{K}}$ in DFT as compared to experiments (see Table I), which leads to an erroneous occupation of both the $\mathrm{K}$ and $\Gamma$ valleys even at very small doping concentrations at room temperature. Something similar, although not as severe, happens also for $\mathrm{WS}_{2}$, for which the DFT prediction for $\Delta E_{\Gamma \mathrm{K}}$ is not much smaller than the experimental results in 
Table I, but it is still sufficient to lead to a spurious softening at large hole doping. Finally, the discrepancy with experiments might also arise from a partial failure of DFT in accounting for band-structure renormalization effects that influence the doping dependence of $\Delta E_{\Gamma \mathrm{K}}$ and $\Delta E_{\mathrm{KQ}}$.

\section{DISCUSSION}

Finding that the strength of the coupling of electrons to phonons is drastically amplified when multiple inequivalent valleys in a given band are populated is a phenomenon that has not been identified in the past, and it is important to build a robust physical understanding. To this end, recall that EPC can be qualitatively understood by considering how the band structure is perturbed by the displacement pattern of the ions associated with the phonon under consideration [4,5,12]. Figure 8 shows the effect of the $\mathrm{A}_{\mathrm{lg}}$ mode on the band structure of undoped monolayer $\mathrm{MoS}_{2}$ (for better clarity, spin-orbit coupling is not included; we checked that this has no qualitative consequences on the arguments below). Although the amplitude (up to $0.12 \AA$ ) of the $\mathrm{A}_{1 \mathrm{~g}}$ mode has been intentionally exaggerated with respect to typical room-temperature mean-square displacements to illustrate this effect, the large variation of both the valence and conduction bands signals the presence of a

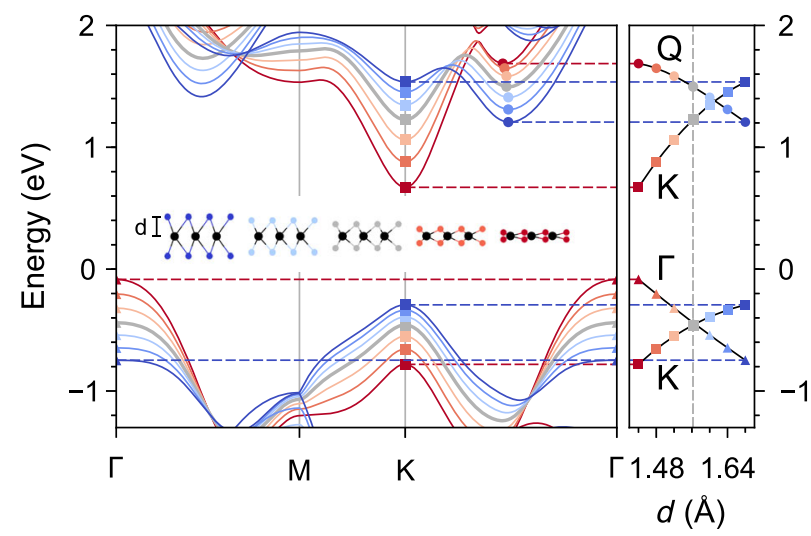

FIG. 8. Variation of the first-principles energy bands of monolayer $\mathrm{MoS}_{2}$ induced by the displacement pattern of the $\mathrm{A}_{1 \mathrm{~g}}$ mode at $\Gamma$ (a rigid vertical motion of the chalcogen planes in opposite directions, with a variation of the distance $d$ between the plane of sulphur atoms and that of molybdenum atoms-see inset). To emphasize the effect, the amplitude of the displacement has been set to $0.12 \AA$, both in compression (red lines) and dilation (blue lines), with respect to the equilibrium value $d=$ $1.57 \AA$ (gray lines). On the right, we report the evolution of the band edges of the K and Q valleys in conduction and of the $\Gamma$ and $\mathrm{K}$ valleys in valence, as a function of $d$. The same colors as in the left panel have been used to identify different values of $d$. The absolute value of the slope of each curve at the equilibrium value of $d$ (marked with a vertical dashed line) is proportional to the bare electron phonon coupling of the corresponding band with the $\mathrm{A}_{1 \mathrm{~g}}$ mode at $\Gamma$, yielding $|g| \approx 50-100 \mathrm{meV}$ depending on the TMD [78-80]. very strong EPC. This strength can be quantified by taking the first derivative of the band energy reported with respect to the atomic displacement, which is proportional to the bare EPC for that band (see the right panel of Fig. 8).

The introduction of free carriers - in our experiments, by means of field-effect doping - allows for the possibility of electrostatic screening and generically tends to decrease the EPC strength (as discussed in Sec. I). In a simple ThomasFermi picture, whether or not the bare EPC is screened effectively by the free carriers depends on whether the evolution of the band induced by the phonon displacement pattern leads to a spatial variation of the density of charge carriers. In this regard, the situation can be very different depending on whether one or more valleys are populated.

To understand why this is the case, we consider a phonon near $\Gamma(\mathbf{q} \rightarrow 0)$ as a frozen atomic displacement with very long, but finite, wavelength. The static displacement associated with such a phonon shifts the valleys up and down in energy locally in space, following the local amplitude of the long-wavelength $A_{1 g}$ mode. In the adiabatic limit, the population of the electronic states will change to keep the system in the lowest energy state. Now, if only a single valley is occupied (say, the $\mathrm{K}$ valley in the conduction band), the only possibility for the free carriers is to follow the spatially varying position of the bottom of the valley to keep the Fermi level constant (or, more appropriately, the electrochemical potential since we are considering the atomic displacement as frozen). This case implies a spatial variation of the charge of the free carriers that self-consistently screens the potential of the lattice displacement, thereby strongly suppressing EPC.

When at least two valleys are occupied, the situation can be very different if the atomic displacements associated with the phonon mode lead to a relative out-of-phase energy shift. This case is indeed so in monolayer TMDs for the $\mathrm{Q}$ and $\mathrm{K}$ valleys in the conduction band and for the $\mathrm{K}$ and $\Gamma$ valleys in the valence band (see Fig. 8). In this case, in the presence of the atomic displacement (and when electrons or holes have time to relax their energy and momentum during the phonon perturbation, i.e., in the adiabatic limit), charge transfer between the valleys must also occur locally in real space for the system to stay in the ground state. Since part of the charge is now involved in this "local" process, the charge that can be displaced to screen the spatially varying potential generated by the phonon is reduced, hindering the effectiveness of screening. In other words, in the presence of two valleys that shift out of phase in response to long-wavelength phonon excitations, screening becomes much less effective in reducing the EPC strength.

The physical picture that we have just qualitatively outlined is based on the results of numerical calculations shown in Appendix C, which are summarized in Fig. 9. In particular, the calculations unambiguously show that the deformation potential at the Fermi level (and thus EPC) is 
completely suppressed because of screening when a single valley is occupied, while it remains finite when multiple valleys are populated. This directly confirms that the correlation between softening and multivalley population emerges from a change in screening of the electron-phonon interaction when free carriers occupy either a single or multiple valleys.

Within the controlled environment of our DFT calculations, we know that this is the mechanism responsible for the strong softening of the $\mathrm{A}_{1 \mathrm{~g}}$ mode in all cases when two valleys are simultaneously occupied. We conclude that this mechanism also explains the Raman measurements, without the need to invoke alternative, more complex scenarios that might seem plausible at first sight. For this mechanism to have a strong effect, it is essential that the phonon mode considered causes an out-of-phase shift in the energy of the two valleys because this maximizes the amount of intervalley charge transfer that occurs locally in real space. For the $\mathrm{E}_{2 \mathrm{~g}}$ mode detected in our Raman measurements on semiconducting TMDs, for instance, this is not the case: The long-range Fröhlich potential associated with the $E_{2 g}$ phonon near $\Gamma$ shifts the energy of the two valleys in the same direction and by comparable amounts so that no (or minimal) intervalley charge transfer occurs locally. As a result, despite the presence of multiple inequivalent valleys, the response of the system to $E_{2 g}$ phonons is similar to the canonical case with one single valley populated, with a strong electrostatic screening that suppresses EPC.

It follows from these considerations that the underlying physical processes responsible for the EPC enhancement in the presence of simultaneously populated inequivalent valleys is of quite general validity. As such, it is likely to occur in a multitude of materials and to have different experimental manifestations. In particular, it could be relevant for ordinary semiconductors such as GaAs-where multiple valleys can be occupied at high doping —although, to our knowledge, it has never been reported before. In this respect, it is quite remarkable that such a mechanism has not been identified earlier. We believe that this is because the analytic theoretical models commonly employed to treat electron-phonon interactions consider only one family of carriers (i.e., one single valley) coupled to phonons, and in these models, the mechanism is simply not present.

Superconductivity provides an example of a physical phenomenon for which the enhanced EPC mechanism that we have identified may be at play. It appears that much of the existing data on gate-induced superconductivity observed in some of the TMDs (e.g., $\mathrm{WS}_{2}$ and $\mathrm{MoS}_{2}$ ) is consistent with having the superconducting instability setting in only when multiple valleys are occupied [25,81]. Having multiple valleys occupied would explain, for instance, why gate-induced superconductivity in $\mathrm{WS}_{2}$ starts occurring at a lower electron density than in $\mathrm{MoS}_{2}$ $[22,24,38]$ because, as we discussed above, the energy difference between the $\mathrm{K}$ and $\mathrm{Q}$ valleys is smaller in $\mathrm{WS}_{2}$ than in $\mathrm{MoS}_{2}$. It also explains why, so far, gate-induced superconductivity in semiconducting TMDs has been observed only upon electron accumulation and not with holes [22,23]: This is because with the hole concentrations that can be reached by field-effect doping, only the $\mathrm{K}$ or $\Gamma$ valleys are filled, so the EPC strength remains weak. Indeed, in $\mathrm{NbSe}_{2}-\mathrm{a}$ metal having a similar valence band structure as $\mathrm{WS}_{2}$ and $\mathrm{MoS}_{2}$, but the Fermi level very deep relative to the top of the valence band $[82,83]$ - robust superconductivity is observed [28-30], and both the $\mathrm{K}$ and the $\Gamma$ valleys are occupied. The multivalley mechanism responsible for the EPC enhancement is likely at play for other superconducting materials, with $\mathrm{MgB}_{2}$ providing a notable example. Indeed, also in $\mathrm{MgB}_{2}$, multiple inequivalent valleys are known to be simultaneously occupied and to move out of phase in energy when atoms are displaced according to a specific phonon pattern [84]. More work is needed to fully substantiate the relevance of multivalley population for the occurrence of superconductivity in all these systems, but the known experimental phenomenology does provide significant circumstantial evidence supporting such a scenario.

\section{ACKNOWLEDGMENTS}

We sincerely thank Francesco Mauri, Matteo Calandra, Haijing Zhang, and Jérémie Teyssier for fruitful discussions. We gratefully acknowledge Alexandre Ferreira for continuous technical support. A. F. M. gratefully acknowledges financial support from the Swiss National Science Foundation (Division II) and from the EU Graphene Flagship project. M. G. and N. U. acknowledge support from the Swiss National Science Foundation through the Ambizione program. Simulation time was provided by CSCS on Piz Daint (Project ID s825) and by PRACE on Marconi at CINECA, Italy (Project ID 2016163963).

\section{APPENDIX A: METHODS}

The realization of the field-effect transistors used to perform the experiments described in the main text relies on a combination of conventional nanofabrication processes and of techniques that are commonly employed to manipulate atomically thin crystals, i.e., 2D materials. Monolayers and bilayers of $\mathrm{MoS}_{2}, \mathrm{WS}_{2}$, and $\mathrm{WSe}_{2}$ are obtained by mechanical exfoliation of bulk crystals and transferred onto $\mathrm{Si} / \mathrm{SiO}_{2}$ substrates. The layer thickness is identified by looking at optical contrast under an optical microscope, and it is confirmed by photoluminescence measurements. To attach electrical contacts to the atomically thin layers, a conventional process based on electron-beam lithography, electron-beam evaporation, and lift-off is used (together with the electrodes, a large side-gate electrode is also deposited). The contacts consist of Pt/Au evaporated films for $\mathrm{WSe}_{2}$ and $\mathrm{Au}$ evaporated films for $\mathrm{WS}_{2}$ and $\mathrm{MoS}_{2}$, and are annealed at $200{ }^{\circ} \mathrm{C}$ in a flow of $\mathrm{Ar}: \mathrm{H}_{2}$ gas 
(100:10 sccm). As a last step, the ionic liquid is drop casted on top of the structure and confined under a 50- $\mu \mathrm{m}$-thick glass plate to ensure a sharp optical image during the optical measurements. As rapidly as possible after depositing the liquid, the device is mounted in the vacuum chamber with optical access (Cryovac KONTI cryostat) where the measurements are performed ( $p \approx 10^{-6}$ mbar). The devices are left under vacuum at least overnight prior to the application of any gate bias to ensure that moisture or oxygen possibly present are pumped out of the system. The ionic liquid used in all experiments is 1-butyl-1methylpyrrolidiniumtris(penta-fluoroethyl) trifluorophosphate [P14][FAP]. All measurements discussed here have been performed at room temperature; at lower temperature, the same procedure cannot be applied because of freezing of the [P14][FAP] ionic liquid.

The Raman scattering spectroscopy measurements are performed using a home-tailored confocal microscope in a backscattering geometry, i.e., collecting the emitted light with the same microscope used to couple the laser beam (at $514.5 \mathrm{~nm}$ ) onto the device [see Fig. 1(a) for a sketch of the experimental setup]. The backscattered light is sent to a Czerni-Turner spectrometer equipped with a 1800-groves/ $\mathrm{mm}$ grating, which resolves the optical spectra with a precision of $0.5 \mathrm{~cm}^{-1}$. The light is detected with the help of a $\mathrm{N}_{2}$-cooled $\mathrm{CCD}$ array (Princeton Instruments). In the experiments, the gate voltage is slowly swept from positive to negative values. At $V_{G}$ intervals of $0.2 \mathrm{~V}$, the sweep is interrupted, and Raman spectra are recorded at fixed $V_{G}$ values. After reaching the largest negative gate voltage, $V_{G}$ is swept back to positive values, and spectra are again recorded at fixed intervals to ensure that the experimental data are free from hysteresis. Finally, we check that the ionic liquid does not possess any characteristic Raman feature in the spectral range of interest.

DFT calculations are performed using Quantum ESPRESSO [85,86], including a cutoff of the Coulomb interaction to implement the correct boundary conditions and gates to simulate doping [21]. We use a symmetric double-gate geometry; for monolayers, a single-gate geometry leads to minimal changes on the results. The exchange-correlation functional is approximated using the Perdew-Burke-Ernzerhof (PBE) form [57] of the generalized-gradient approximation. To compute full phonon dispersions, we use the SSSP Accuracy library [87] (version 0.7), a 60-Ry energy cutoff, and a $32 \times 32 \times 1$ grid with 0.02-Ry Marzari-Vanderbilt broadening [74]. We then use density-functional perturbation theory [72] including Coulomb cutoff and gates-to compute the phonons on a $12 \times 12 \times 1$ grid in the Brillouin zone. For zone-center phonon calculations, we significantly reduce smearing and increase the sampling accordingly: Namely, we use a dense $120 \times 120 \times 1 k$-point grid and Fermi-Dirac smearing corresponding to room temperature $(0.002 \mathrm{Ry})$. Spin-orbit interaction is included by using fully relativistic norm-conserving pseudopotentials from the Pseudo-Dojo library [88]. Phonopy [89] is then used to generate the atomic-displacement patterns and to postprocess forces to obtain phonon frequencies.

\section{APPENDIX B: JUSTIFICATION OF EQ. (1) IN THE MAIN TEXT}

In the following, we show how to use expressions derived in Refs. [68-70] to obtain Eq. (1) with the help of a few assumptions valid in the case of semiconducting TMDs. We are interested in the doping-induced frequency variations of a phonon mode at $\Gamma$ (phonon momentum $\mathbf{q} \rightarrow 0$ ) with respect to its value in the neutral case $\Delta \omega=$ $\omega_{\text {doped }}-\omega_{\text {neutral }}$. The frequency of a given phonon mode corresponding to an eigenvector $|\varepsilon\rangle$ of the dynamical matrix at $\Gamma, \mathcal{D}(\mathbf{q}=0)$, can be obtained as $\omega^{2}=$ $\langle\varepsilon|\mathcal{D}(\mathbf{q}=0)| \varepsilon\rangle /\langle\varepsilon \mid \varepsilon\rangle=\mathcal{D}_{\Gamma} / M$, where the eigenvectors are defined such that $M=\langle\varepsilon \mid \varepsilon\rangle$ for convenience, $M$ being an effective mass associated with the phonon mode and $\mathcal{D}_{\Gamma}=\langle\varepsilon|\mathcal{D}(\mathbf{q}=0)| \varepsilon\rangle=\langle u|\mathcal{C}(\mathbf{q}=0)| u\rangle$ is the projection of the dynamical matrix on the corresponding eigenvector or, equivalently, of the force constants $\mathcal{C}$ on the physical displacements $|u\rangle$ of the phonon mode (with $\langle u \mid u\rangle=1$ ). Assuming that the variation of the frequency is small compared to the neutral frequency $\Delta \omega \ll \omega_{\text {neutral, }}$, we can differentiate $\left(d \omega \approx d \omega^{2} / 2 \omega\right)$ to obtain the following simple relation:

$$
\Delta \omega \approx \frac{\Delta \mathcal{D}_{\Gamma}}{2 M \omega_{\text {neutral }}} .
$$

Neglecting the variations with doping of the contributions from the second-order derivatives of the ionic potential and the exchange-correlation kernel with respect to the atomic displacements in Eq. (3) of Ref. [69], we write

$$
\Delta \mathcal{D}_{\Gamma} \approx \Delta \mathcal{F}_{\Gamma}
$$

where $\mathcal{F}_{\Gamma}$ is the part of the dynamical matrix at the $\Gamma$ point involving the electronic response to the phonon perturbation and thus electron-phonon interactions. The explicit expression for $\mathcal{F}_{\Gamma}$ depends on the adiabaticity of the system. In the adiabatic limit relevant for TMDs, it reads [68-70]

$$
\begin{gathered}
\mathcal{F}_{\Gamma}=\frac{1}{N_{\mathbf{k}}} \sum_{\mathbf{k}, n \neq m}\left|\left\langle\mathbf{k} m\left|\Delta V_{\Gamma, \nu}\right| \mathbf{k} n\right\rangle\right|^{2} \frac{f_{\mathbf{k}, m}-f_{\mathbf{k}, n}}{\varepsilon_{\mathbf{k}, m}-\varepsilon_{\mathbf{k}, n}} \\
+\left.\frac{1}{N_{\mathbf{k}}} \sum_{\mathbf{k}, n}\left|\left\langle\mathbf{k} n\left|\Delta V_{\Gamma, \nu}\right| \mathbf{k} n\right\rangle\right|^{2} \frac{\partial f}{\partial \varepsilon}\right|_{\varepsilon_{\mathbf{k}, n}},
\end{gathered}
$$

where $\Delta V_{\mathbf{q}, \nu}$ is the perturbation of the effective potential due to phonon $(\mathbf{q}, \nu)$, and $f_{\mathbf{k}, n}$ is the occupation of the state 
$|\mathbf{k} n\rangle$ corresponding to the band $n$ at crystal momentum $\mathbf{k}$ with energy $\varepsilon_{\mathbf{k}, n}$. In the first term, there are only interband contributions $(n \neq m)$ between occupied and empty states. We have verified that the numerator is much smaller than the denominator $\left|\varepsilon_{\mathbf{k}, m}-\varepsilon_{\mathbf{k}, n}\right|$, which is equal to the direct gap in the neutral case, so this term can be neglected. The second term involves intraband transitions between states close to the Fermi surface, and it is nonzero only in doped systems and vanishing in neutral TMDs. This result is the main contribution to doping-induced variations, so we can write

$$
\begin{aligned}
\Delta \omega & \left.\approx \frac{\Delta \mathcal{F}_{\Gamma}}{2 M \omega_{\text {neutral }}} \approx \frac{1}{N_{\mathbf{k}}} \sum_{\mathbf{k}, n} \frac{\left|\left\langle\mathbf{k} n\left|\Delta V_{\Gamma, \nu}\right| \mathbf{k} n\right\rangle\right|^{2}}{2 M \omega_{\text {neutral }}} \frac{\partial f}{\partial \varepsilon}\right|_{\varepsilon_{\mathbf{k}, n}} \\
& =\left.\frac{1}{N_{\mathbf{k}}} \sum_{\mathbf{k}, n}\left|g_{\nu, \mathbf{k}, \mathbf{k}}^{(n)}\right|^{2} \frac{\partial f}{\partial \varepsilon}\right|_{\varepsilon_{\mathbf{k}, n}},
\end{aligned}
$$

where we have introduced the square electron-phonon matrix element due to intraband transitions:

$$
\left|g_{\nu, \mathbf{k}, \mathbf{k}}^{(n)}\right|^{2}=\frac{\left|\left\langle\mathbf{k} n\left|\Delta V_{\Gamma, \nu}\right| \mathbf{k} n\right\rangle\right|^{2}}{2 M \omega_{\text {neutral }}} .
$$

The derivative of the Fermi distribution is negative (the phonon frequency softens) and nonzero only in a small energy window of order $k_{B} T$ around the chemical potential, so only electronic states belonging to the temperaturesmeared Fermi surface contribute. In the zero-temperature limit, the derivative becomes a delta function at the Fermi energy $\varepsilon_{F}$, and we obtain

$$
\begin{aligned}
\Delta \omega & \approx-\frac{1}{N_{\mathbf{k}}} \sum_{\mathbf{k}, n}\left|g_{\nu, \mathbf{k}, \mathbf{k}}^{(n)}\right|^{2} \delta\left(\varepsilon_{\mathbf{k}, n}-\varepsilon_{F}\right) \\
& =-N\left(\varepsilon_{F}\right)\left\langle g_{\nu}^{2}\right\rangle_{\mathrm{FS}},
\end{aligned}
$$

which is Eq. (1) of the main text. Here, we have introduced the density of states at the Fermi energy,

$$
N\left(\varepsilon_{F}\right)=\frac{1}{N_{\mathbf{k}}} \sum_{\mathbf{k}, n} \delta\left(\varepsilon_{\mathbf{k}, n}-\varepsilon_{F}\right),
$$

and the square EPC averaged over the Fermi surface:

$$
\left\langle g_{\nu}^{2}\right\rangle_{\mathrm{FS}}=\frac{\sum_{\mathbf{k}, n}\left|g_{\nu, \mathbf{k}, \mathbf{k}}^{(n)}\right|^{2} \delta\left(\varepsilon_{\mathbf{k}, n}-\varepsilon_{F}\right)}{\sum_{\mathbf{k}, n} \delta\left(\varepsilon_{\mathbf{k}, n}-\varepsilon_{F}\right)} .
$$

\section{APPENDIX C: EFFECT OF MULTIVALLEY POPULATIONS ON SCREENING OF THE ELECTRON-PHONON INTERACTION}

According to the theory of deformation potentials [4,5], the coupling of an electron in a given band state at momentum $\mathbf{k}$ with a long-wavelength phonon is proportional to the change in energy of the state with the amplitude of the phonon displacement. Considering, for definiteness, the case of the $\mathrm{A}_{1 \mathrm{~g}}$ mode, this means that

$$
\left|g_{A_{1 g}, \mathbf{k}, \mathbf{k}}\right| \propto\left|\frac{\partial \varepsilon_{\mathbf{k}}}{\partial d}\right|,
$$

where $\varepsilon_{\mathbf{k}}$ is the energy of the state at $\mathbf{k}$ and we have parametrized the amplitude of the $\mathrm{A}_{1 \mathrm{~g}}$ with the vertical separation $d$ of the plane of chalcogen atoms with respect to the plane of transition metal atoms (see also Figs. 1 and 8).

When computing the EPC using Eq. (C1), it should be kept in mind that the energy of a state in a periodic solid has no absolute meaning, and it needs to be specified with respect to a well-defined reference value. This idea is particularly relevant in the evaluation of deformation potentials, as one needs to compare the energy of a state in different calculations. In the calculations presented here, performed in metallic systems at fixed charge and in the adiabatic limit, the appropriate reference value is the electrochemical potential [12].

In Fig. 9, we report the band structure of monolayer $\mathrm{MoS}_{2}$ as a function of the amplitude of the $\mathrm{A}_{1 \mathrm{~g}}$ mode and for two different doping levels: (a) low doping, when only the $\mathrm{K}$ valley is occupied, and (b) high doping, when both the $\mathrm{K}$ and the $\mathrm{Q}$ valleys are occupied. The difference with respect to Fig. 8 in the main text for the neutral case is that we are now referring the band energies to the electrochemical potential of the doped system. The deformation potentials computed this way implicitly assume adiabaticity and include perfect metallic screening from the charge carriers added in the simulations.

For both doping levels, on the right, we report the energy of a state close to the Fermi surface as a function of $d$. According to Eq. (C1), the slope of this curve gives a measure of the EPC at the Fermi surface and thus, through Eq. (1), to the softening of the $A_{1 g}$ mode. These plots clearly show that the deformation potential at the Fermi surface vanishes when only one valley $(\mathrm{K})$ is occupied, while it is significant when two types of valleys are occupied and move out of phase with the phonon perturbation. This case is consistent with the observation that softening emerges only when two valleys are occupied.

These results allow us to also investigate the effect of free-carrier screening by comparing Fig. 9, where a finite density of free carriers is considered, with Fig. 8 for the neutral case, where free carriers are not present and cannot screen. We first note that the large bare deformation potential of the $\mathrm{K}$ valley visible in Fig. 8 is greatly suppressed when free carriers are introduced and populate only one valley [Fig. 9(a)]. This result is from screening by free carriers, which requires both the electrochemical potential and the charge to stay constant as a function of the phonon amplitude, leaving the energy of the states close to the Fermi surface almost unaffected by the phonon 

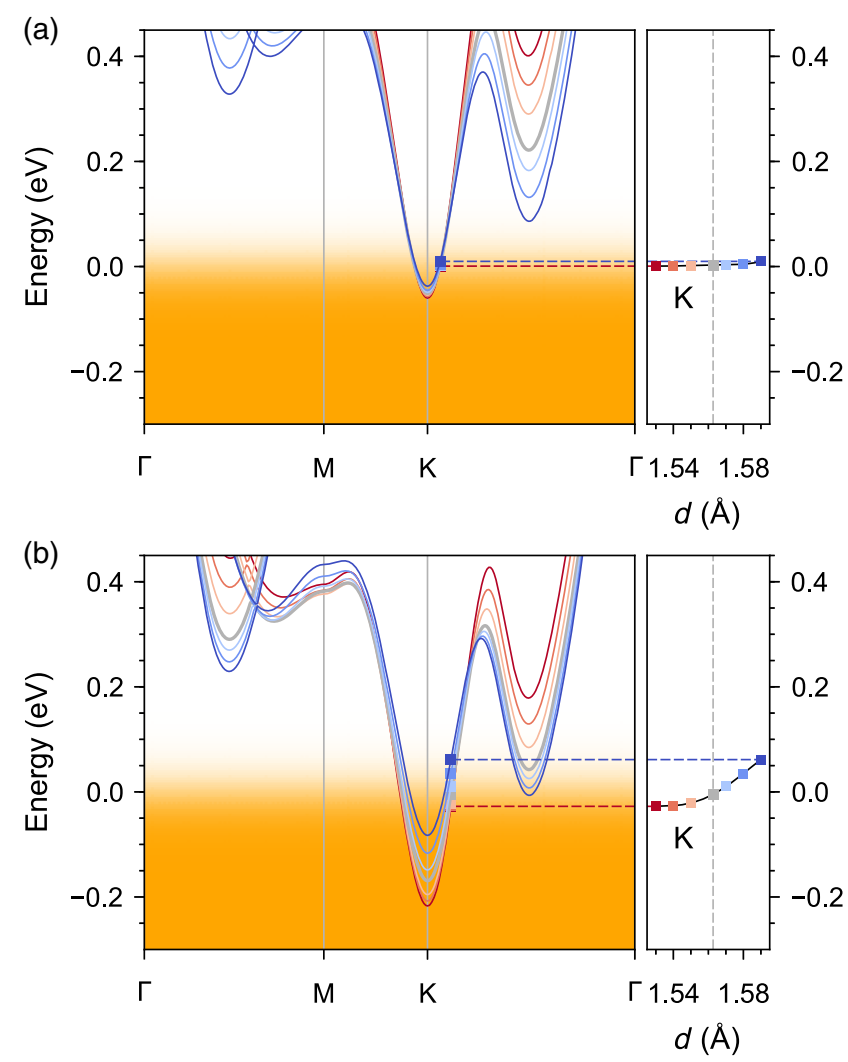

FIG. 9. Variation of the conduction band energies in electrondoped monolayer $\mathrm{MoS}_{2}$ as a function of the amplitude of the $\mathrm{A}_{1 \mathrm{~g}}$ mode parametrized with the vertical separation $d$ of the plane of chalcogen atoms with respect to the plane of transition metal atoms. The electrochemical potential $\mu$ is used as a reference. Electronic states are occupied according to a Fermi-Dirac distribution at $300 \mathrm{~K}$, shown as a color shading (dark yellow corresponding to 1 and white to 0 ). Two different doping levels are considered: (a) low doping ( $n=0.02 \mathrm{e} / \mathrm{cell}$ ), when only the $\mathrm{K}$ valley is occupied, and (b) high doping ( $n=0.08 e /$ cell), when both $\mathrm{K}$ and $\mathrm{Q}$ valleys are populated.

perturbation. On the contrary, when both the $\mathrm{K}$ and $\mathrm{Q}$ valleys are occupied [Fig. 9(b)], a transfer of the electronic population from one valley to the other is possible as a result of the out-of-phase shift of the valleys, so at least in part the phonon perturbation can happen without changes in the local charge, making it insensitive to screening from free carriers. This case results in a deformation potential that is nonzero and very close to the bare value in the undoped system (Fig. 8).

We can thus conclude that the correlation between softening and multivalley populations is also manifest when directly computing the EPC through deformation potentials, and that the effect is due to a difference in screening by free carriers when one or multiple valleys are occupied.

[1] J. M. Ziman, Electrons and Phonons: The Theory of Transport Phenomena in Solids (Clarendon, Oxford, 1960).
[2] G. Grimvall, The Electron-Phonon Interaction in Metals (North-Holland, Amsterdam, 1981).

[3] G. D. Mahan, Many-Particle Physics, 3rd ed. (Plenum, New York, 2000).

[4] J. Bardeen and W. Shockley, Deformation Potentials and Mobilities in Non-Polar Crystals, Phys. Rev. 80, 72 (1950).

[5] W. Shockley and J. Bardeen, Energy Bands and Mobilities in Monatomic Semiconductors, Phys. Rev. 77, 407 (1950).

[6] L. Pietronero, S. Strässler, H. R. Zeller, and M. J. Rice, Electrical Conductivity of a Graphite Layer, Phys. Rev. B 22, 904 (1980).

[7] T. Sohier, M. Calandra, C.-H. Park, N. Bonini, N. Marzari, and F. Mauri, Phonon-Limited Resistivity of Graphene by First-Principles Calculations: Electron-Phonon Interactions, Strain-Induced Gauge Field, and Boltzmann Equation, Phys. Rev. B 90, 125414 (2014).

[8] M. Vozmediano, M. Katsnelson, and F. Guinea, Gauge Fields in Graphene, Phys. Rep. 496, 109 (2010).

[9] S. Engelsberg and J.R. Schrieffer, Coupled ElectronPhonon System, Phys. Rev. 131, 993 (1963).

[10] E. Maksimov and S. Shulga, Nonadiabatic Effects in Optical Phonon Self-Energy, Solid State Commun. 97, 553 (1996).

[11] P. Bogulsawski and J. Mycielski, Is the Deformation Potential in Semiconductors Screened by Free Carriers?, J. Phys. C 10, 2413 (1977).

[12] F. S. Khan and P. B. Allen, Deformation Potentials and Electron-Phonon Scattering: Two New Theorems, Phys. Rev. B 29, 3341 (1984).

[13] E. Kartheuser and S. Rodriguez, Deformation Potentials and the Electron-Phonon Interaction in Metals, Phys. Rev. B 33, 772 (1986).

[14] A.C. Ferrari, Raman Spectroscopy of Graphene and Graphite: Disorder, Electron-Phonon Coupling, Doping and Nonadiabatic Effects, Solid State Commun. 143, 47 (2007).

[15] A. C. Ferrari and D. M. Basko, Raman Spectroscopy as a Versatile Tool for Studying the Properties of Graphene, Nat. Nanotechnol. 8, 235 (2013).

[16] S. Pisana, M. Lazzeri, C. Casiraghi, K. S. Novoselov, A. K. Geim, A.C. Ferrari, and F. Mauri, Breakdown of the Adiabatic Born-Oppenheimer Approximation in Graphene, Nat. Mater. 6, 198 (2007).

[17] D. A. Kirzhnits, E. G. Maksimov, and D. I. Khomskii, The Description of Superconductivity in Terms of Dielectric Response Function, J. Low Temp. Phys. 10, 79 (1973).

[18] J. L. M. van Mechelen, D. van der Marel, C. Grimaldi, A. B. Kuzmenko, N. P. Armitage, N. Reyren, H. Hagemann, and I. I. Mazin, Electron-Phonon Interaction and Charge Carrier Mass Enhancement in $\mathrm{SrTiO}_{3}$, Phys. Rev. Lett. 100, 226403 (2008).

[19] Z. Wang et al., Tailoring the Nature and Strength of Electron-Phonon Interactions in the $\mathrm{SrTiO}_{3}(001) 2 \mathrm{D}$ Electron Liquid, Nat. Mater. 15, 835 (2016).

[20] C. Verdi, F. Caruso, and F. Giustino, Origin of the Crossover from Polarons to Fermi Liquids in Transition Metal Oxides, Nat. Commun. 8, 15769 (2017).

[21] T. Sohier, M. Calandra, and F. Mauri, Density Functional Perturbation Theory for Gated Two-Dimensional Heterostructures: Theoretical Developments and Application to 
Flexural Phonons in Graphene, Phys. Rev. B 96, 075448 (2017).

[22] J. T. Ye, Y. J. Zhang, R. Akashi, M. S. Bahramy, R. Arita, and Y. Iwasa, Superconducting Dome in a Gate-Tuned Band Insulator, Science 338, 1193 (2012).

[23] S. Jo, D. Costanzo, H. Berger, and A. F. Morpurgo, Electrostatically Induced Superconductivity at the Surface of $\mathrm{WS}_{2}$, Nano Lett. 15, 1197 (2015).

[24] J. Lu, O. Zheliuk, Q. Chen, I. Leermakers, N. E. Hussey, U. Zeitler, and J. Ye, Full Superconducting Dome of Strong Ising Protection in Gated Monolayer $\mathrm{WS}_{2}$, Proc. Natl. Acad. Sci. U.S.A. 115, 3551 (2018).

[25] E. Piatti, D. De Fazio, D. Daghero, S. R. Tamalampudi, D. Yoon, A. C. Ferrari, and R. S. Gonnelli, Multi-Valley Superconductivity in Ion-Gated $\mathrm{MoS}_{2}$ Layers, Nano Lett. 18, 4821 (2018).

[26] Y. Hamaue and R. Aoki, Effects of Organic Intercalation on Lattice Vibrations and Superconducting Properties of $2 \mathrm{H}-\mathrm{NbS}_{2}$, J. Phys. Soc. Jpn. 55, 1327 (1986).

[27] I. Guillamón, H. Suderow, S. Vieira, L. Cario, P. Diener, and P. Rodière, Superconducting Density of States and Vortex Cores of $2 \mathrm{H}-\mathrm{NbS}_{2}$, Phys. Rev. Lett. 101, 166407 (2008).

[28] X. Xi, Z. Wang, W. Zhao, J.-H. Park, K. T. Law, H. Berger, L. Forró, J. Shan, and K. F. Mak, Ising Pairing in Superconducting $\mathrm{NbSe}_{2}$ Atomic Layers, Nat. Phys. 12, 139 (2016).

[29] M. M. Ugeda, A. J. Bradley, Y. Zhang, S. Onishi, Y. Chen, W. Ruan, C. Ojeda-Aristizabal, H. Ryu, M. T. Edmonds, H.-Z. Tsai, A. Riss, S.-K. Mo, D. Lee, A. Zettl, Z. Hussain, Z.-X. Shen, and M. F. Crommie, Characterization of Collective Ground States in Single-Layer $\mathrm{NbSe}_{2}$, Nat. Phys. 12, 92 (2016).

[30] A. W. Tsen, B. Hunt, Y. D. Kim, Z. J. Yuan, S. Jia, R. J. Cava, J. Hone, P. Kim, C. R. Dean, and A. N. Pasupathy, Nature of the Quantum Metal in a Two-Dimensional Crystalline Superconductor, Nat. Phys. 12, 208 (2016).

[31] D. Braga, I. G. Lezama, H. Berger, and A. F. Morpurgo, Quantitative Determination of the Band Gap of $\mathrm{WS}_{2}$ with Ambipolar Ionic Liquid-Gated Transistors, Nano Lett. 12, 5218 (2012).

[32] Y. J. Zhang, T. Oka, R. Suzuki, J. T. Ye, and Y. Iwasa, Electrically Switchable Chiral Light-Emitting Transistor, Science 344, 725 (2014).

[33] I. Gutiérrez-Lezama, A. Ubaldini, M. Longobardi, E. Giannini, C. Renner, A. B. Kuzmenko, and A. F. Morpurgo, Surface Transport and Band Gap Structure of Exfoliated 2H-MoTe ${ }_{2}$ Crystals, 2D Mater. 1, 021002 (2014).

[34] S. Jo, N. Ubrig, H. Berger, A. B. Kuzmenko, and A. F. Morpurgo, Mono- and Bilayer $\mathrm{WS}_{2}$ Light-Emitting Transistors, Nano Lett. 14, 2019 (2014).

[35] N. Ubrig, S. Jo, H. Berger, A. F. Morpurgo, and A. B. Kuzmenko, Scanning Photocurrent Microscopy Reveals Electron-Hole Asymmetry in Ionic Liquid-Gated $\mathrm{WS}_{2}$ Transistors, Appl. Phys. Lett. 104, 171112 (2014).

[36] E. Ponomarev, I. Gutiérrez-Lezama, N. Ubrig, and A. F. Morpurgo, Ambipolar Light-Emitting Transistors on Chemical Vapor Deposited Monolayer $\mathrm{MoS}_{2}$, Nano Lett. 15, 8289 (2015).

[37] Y. Zhang, J. Ye, Y. Matsuhashi, and Y. Iwasa, Ambipolar $\mathrm{MoS}_{2}$ Thin Flake Transistors, Nano Lett. 12, 1136 (2012).
[38] D. Costanzo, S. Jo, H. Berger, and A. F. Morpurgo, GateInduced Superconductivity in Atomically Thin $\mathrm{MoS}_{2}$ Crystals, Nat. Nanotechnol. 11, 339 (2016).

[39] I. Gutiérrez-Lezama, B. A. Reddy, N. Ubrig, and A. F. Morpurgo, Electroluminescence from Indirect Band Gap Semiconductor $\mathrm{ReS}_{2}$, 2D Mater. 3, 045016 (2016).

[40] C. Lee, H. Yan, L. E. Brus, T. F. Heinz, J. Hone, and S. Ryu, Anomalous Lattice Vibrations of Single- and Few-Layer $\mathrm{MoS}_{2}$, ACS Nano 4, 2695 (2010).

[41] A. Molina-Sánchez and L. Wirtz, Phonons in Single-Layer and Few-Layer $\mathrm{MoS}_{2}$ and $\mathrm{WS}_{2}$, Phys. Rev. B 84, 155413 (2011).

[42] A. Berkdemir, H. R. Gutiérrez, A. R. Botello-Méndez, N. Perea-López, A. L. Elías, C.-I. Chia, B. Wang, V. H. Crespi, F. López-Urías, J.-C. Charlier, H. Terrones, and M. Terrones, Identification of Individual and Few Layers of $\mathrm{WS}_{2}$ Using Raman Spectroscopy, Sci. Rep. 3, 1755 (2013).

[43] H. Sahin, S. Tongay, S. Horzum, W. Fan, J. Zhou, J. Li, J. $\mathrm{Wu}$, and F. M. Peeters, Anomalous Raman Spectra and Thickness-Dependent Electronic Properties of $\mathrm{WSe}_{2}$, Phys. Rev. B 87, 165409 (2013).

[44] R. Saito, Y. Tatsumi, S. Huang, X. Ling, and M. S. Dresselhaus, Raman Spectroscopy of Transition Metal Dichalcogenides, J. Phys. Condens. Matter 28, 353002 (2016).

[45] H. Terrones, E. D. Corro, S. Feng, J. M. Poumirol, D. Rhodes, D. Smirnov, N. R. Pradhan, Z. Lin, M. a. T. Nguyen, A. L. Elías, T. E. Mallouk, L. Balicas, M. A. Pimenta, and M. Terrones, New First Order Raman-Active Modes in Few Layered Transition Metal Dichalcogenides, Sci. Rep. 4, 4215 (2014).

[46] A. A. Mitioglu, P. Plochocka, G. Deligeorgis, S. Anghel, L. Kulyuk, and D. K. Maude, Second-Order Resonant Raman Scattering in Single-Layer Tungsten Disulfide $\mathrm{WS}_{2}$, Phys. Rev. B 89, 245442 (2014).

[47] A. Carvalho, R. M. Ribeiro, and A. H. C. Neto, Band Nesting and the Optical Response of Two-Dimensional Semiconducting Transition Metal Dichalcogenides, Phys. Rev. B 88, 115205 (2013).

[48] The values of the carrier density are estimated using values of the capacitance per unit area obtained from previous experiments (in which the capacitance was obtained using the Hall effect to measure the density of accumulated charge). These past experiments show that in ionic-liquid gated transistors on monolayer and bilayer semiconducting TMDS - in the range of density explored here - the capacitance value is largely determined by the density of states (i.e., taking into account the quantum capacitance is essential). Since the density of states is different for monolayers and bilayers and — once the thickness is fixed-for the conduction and valence bands, different values for $C$ have to be used. At room temperature, the capacitance does not depend much on the specific compound considered, and here we use the values measured on $\mathrm{WSe}_{2}$ monolayers and bilayers, which are $\mathrm{C}_{\mathrm{ML}}^{e}=7.3 \mu \mathrm{F} \mathrm{cm}^{-2}, \mathrm{C}_{\mathrm{ML}}^{h}=17 \mu \mathrm{F} \mathrm{cm}^{-2}, \mathrm{C}_{\mathrm{BL}}^{e}=$ $22.8 \mu \mathrm{F} \mathrm{cm}^{-2}, \mathrm{C}_{\mathrm{BL}}^{h}=24 \mu \mathrm{F} \mathrm{cm}^{-2}$ (the subscripts $\mathrm{ML}$ and $\mathrm{BL}$ refer to monolayer and bilayer; the superscripts $e$ and $h$ indicate the value to use for accumulation of electrons and holes, respectively). 
[49] In view of the microscopic complexity of the resonant process from which the $2 \mathrm{LA}(\mathrm{M})$ Raman peak originates, and of the fact that-as will be discussed explicitly in Sec. III-our main interest here is on long-wavelength phonons, we will not discuss the behavior of the 2LA(M) Raman peak. As an only remark, note that-just like for the $A_{1 \mathrm{~g}}$ mode - the LA(M) mode corresponds to an out-of-plane motion of the atoms in the lattice [see Fig. 1(c)], and a finite EPC is present, as demonstrated by the sensitivity of the mode frequency to the introduction of charge carriers.

[50] B. Chakraborty, A. Bera, D. V. S. Muthu, S. Bhowmick, U. V. Waghmare, and A. K. Sood, Symmetry-Dependent Phonon Renormalization in Monolayer $\mathrm{MoS}_{2}$ Transistor, Phys. Rev. B 85, 161403(R) (2012).

[51] X. Lu, M. I. B. Utama, X. Wang, W. Xu, W. Zhao, M. H. S. Owen, and Q. Xiong, Gate-Tunable Resonant Raman Spectroscopy of Bilayer $\mathrm{MoS}_{2}$, Small 13, 1701039 (2017).

[52] A. Molina-Sánchez, K. Hummer, and L. Wirtz, Vibrational and Optical Properties of $\mathrm{MoS}_{2}$ : From Monolayer to Bulk, Surf. Sci. Rep. 70, 554 (2015).

[53] D. Le, A. Barinov, E. Preciado, M. Isarraraz, I. Tanabe, T. Komesu, C. Troha, L. Bartels, T. S. Rahman, and P. A. Dowben, Spin-Orbit Coupling in the Band Structure of Monolayer $\mathrm{WSe}_{2}$, J. Phys. Condens. Matter 27, 182201 (2015).

[54] A. Kormányos, G. Burkard, M. Gmitra, J. Fabian, V. Zólyomi, N. D. Drummond, and V. Fal'ko, k·p Theory for Two-Dimensional Transition Metal Dichalcogenide Semiconductors, 2D Mater. 2, 022001 (2015).

[55] H. Yuan et al., Evolution of the Valley Position in Bulk Transition-Metal Chalcogenides and Their Monolayer Limit, Nano Lett. 16, 4738 (2016).

[56] L. Ortenzi, L. Pietronero, and E. Cappelluti, Zero-Point Motion and Direct-Indirect Band-Gap Crossover in Layered Transition-Metal Dichalcogenides, Phys. Rev. B 98, 195313 (2018).

[57] J. P. Perdew, K. Burke, and M. Ernzerhof, Generalized Gradient Approximation Made Simple, Phys. Rev. Lett. 77, 3865 (1996).

[58] W. Jin, P.-C. Yeh, N. Zaki, D. Zhang, J. T. Sadowski, A. AlMahboob, A. M. van der Zande, D. A. Chenet, J. I. Dadap, I. P. Herman, P. Sutter, J. Hone, and R. M. Osgood, Direct Measurement of the Thickness-Dependent Electronic Band Structure of $\mathrm{MoS}_{2}$ Using Angle-Resolved Photoemission Spectroscopy, Phys. Rev. Lett. 111, 106801 (2013).

[59] J. A. Miwa, S. Ulstrup, S. G. Sørensen, M. Dendzik, A. G. Cabo, M. Bianchi, J. V. Lauritsen, and P. Hofmann, Electronic Structure of Epitaxial Single-Layer $\mathrm{MoS}_{2}$, Phys. Rev. Lett. 114, 046802 (2015).

[60] P. V. Nguyen, N. C. Teutsch, N. P. Wilson, J. Kahn, X. Xia, V. Kandyba, A. Barinov, G. Constantinescu, N. D. Hine, X. $\mathrm{Xu}$ et al., Visualizing Electrostatic Gating Effects in TwoDimensional Heterostructures, arXiv:1904.07301.

[61] M. Dendzik, M. Michiardi, C. Sanders, M. Bianchi, J. A. Miwa, S. S. Grønborg, J. V. Lauritsen, A. Bruix, B. Hammer, and P. Hofmann, Growth and Electronic Structure of Epitaxial Single-Layer $\mathrm{WS}_{2}$ on Au(111), Phys. Rev. B 92 , 245442 (2015).

[62] C. Kastl, C. T. Chen, R. J. Koch, B. Schuler, T. R. Kuykendall, A. Bostwick, C. Jozwiak, T. Seyller,
E. Rotenberg, A. Weber-Bargioni, S. Aloni, and A. M. Schwartzberg, Multimodal Spectromicroscopy of Monolayer $\mathrm{WS}_{2}$ Enabled by Ultra-clean van der Waals Epitaxy, 2D Mater. 5, 045010 (2018).

[63] H. Henck, Z. B. Aziza, D. Pierucci, F. Laourine, F. Reale, P. Palczynski, J. Chaste, M. G. Silly, F. Bertran, P. Le Fèvre, E. Lhuillier, T. Wakamura, C. Mattevi, J. E. Rault, M. Calandra, and A. Ouerghi, Electronic Band Structure of Two-Dimensional $\mathrm{WS}_{2}$ /Graphene van der Waals Heterostructures, Phys. Rev. B 97, 155421 (2018).

[64] Y. Zhang, M. M. Ugeda, C. Jin, S.-F. Shi, A. J. Bradley, A. Martín-Recio, H. Ryu, J. Kim, S. Tang, Y. Kim, B. Zhou, C. Hwang, Y. Chen, F. Wang, M. F. Crommie, Z. Hussain, Z.-X. Shen, and S.-K. Mo, Electronic Structure, Surface Doping, and Optical Response in Epitaxial $\mathrm{WSe}_{2}$ Thin Films, Nano Lett. 16, 2485 (2016).

[65] N. R. Wilson, P. V. Nguyen, K. Seyler, P. Rivera, A. J. Marsden, Z. P. L. Laker, G. C. Constantinescu, V. Kandyba, A. Barinov, N. D. M. Hine, X. Xu, and D. H. Cobden, Determination of Band Offsets, Hybridization, and Exciton Binding in 2D Semiconductor Heterostructures, Sci. Adv. 3, e1601832 (2017).

[66] Z. Y. Zhu, Y. C. Cheng, and U. Schwingenschlögl, Giant Spin-Orbit-Induced Spin Splitting in Two-Dimensional Transition-Metal Dichalcogenide Semiconductors, Phys. Rev. B 84, 153402 (2011).

[67] M. Gibertini, F. M. D. Pellegrino, N. Marzari, and M. Polini, Spin-Resolved Optical Conductivity of Two-Dimensional Group-VIB Transition-Metal Dichalcogenides, Phys. Rev. B 90, 245411 (2014).

[68] M. Lazzeri and F. Mauri, Nonadiabatic Kohn Anomaly in a Doped Graphene Monolayer, Phys. Rev. Lett. 97, 266407 (2006).

[69] A. M. Saitta, M. Lazzeri, M. Calandra, and F. Mauri, Giant Nonadiabatic Effects in Layer Metals: Raman Spectra of Intercalated Graphite Explained, Phys. Rev. Lett. 100, 226401 (2008).

[70] M. Calandra, G. Profeta, and F. Mauri, Adiabatic and Nonadiabatic Phonon Dispersion in a Wannier Function Approach, Phys. Rev. B 82, 165111 (2010).

[71] F. Marsiglio, R. Akis, and J. P. Carbotte, Phonon SelfEnergy Effects Due to Superconductivity: A Real-Axis Formulation, Phys. Rev. B 45, 9865 (1992).

[72] S. Baroni, S. de Gironcoli, A. Dal Corso, and P. Giannozzi, Phonons and Related Crystal Properties from DensityFunctional Perturbation Theory, Rev. Mod. Phys. 73, 515 (2001).

[73] M. Kang, S. W. Jung, W. J. Shin, Y. Sohn, S. H. Ryu, T. K. Kim, M. Hoesch, and K. S. Kim, Holstein Polaron in a Valley-Degenerate Two-Dimensional Semiconductor, Nat. Mater. 17, 676 (2018).

[74] N. Marzari, D. Vanderbilt, A. De Vita, and M. C. Payne, Thermal Contraction and Disordering of the Al(110) Surface, Phys. Rev. Lett. 82, 3296 (1999).

[75] S. Gao and L. Yang, Renormalization of the Quasiparticle Band Gap in Doped Two-Dimensional Materials from Many-Body Calculations, Phys. Rev. B 96, 155410 (2017).

[76] J. Katoch, S. Ulstrup, R. J. Koch, S. Moser, K. M. McCreary, S. Singh, J. Xu, B. T. Jonker, R. K. Kawakami, A. Bostwick, E. Rotenberg, and C. Jozwiak, Giant 
Spin-Splitting and Gap Renormalization Driven by Trions in Single-Layer $\mathrm{WS}_{2} / \mathrm{h}-\mathrm{BN}$ Heterostructures, Nat. Phys. 14, 355 (2018).

[77] M. Kang, B. Kim, S. H. Ryu, S. W. Jung, J. Kim, L. Moreschini, C. Jozwiak, E. Rotenberg, A. Bostwick, and K. S. Kim, Universal Mechanism of Band-Gap Engineering in Transition-Metal Dichalcogenides, Nano Lett. 17, 1610 (2017).

[78] Z. Jin, X. Li, J. T. Mullen, and K. W. Kim, Intrinsic Transport Properties of Electrons and Holes in Monolayer Transition-Metal Dichalcogenides, Phys. Rev. B 90, 045422 (2014).

[79] X. Li, J. T. Mullen, Z. Jin, K. M. Borysenko, M. B. Nardelli, and K. W. Kim, Intrinsic Electrical Transport Properties of Monolayer Silicene and $\mathrm{MoS}_{2}$ from First Principles, Phys. Rev. B 87, 115418 (2013).

[80] T. Sohier, D. Campi, N. Marzari, and M. Gibertini, Mobility of 2D Materials from First Principles in an Accurate and Automated Framework, Phys. Rev. Mater. 2, 114010 (2018).

[81] E. Piatti, D. Romanin, and R. S. Gonnelli, Mapping Multivalley Lifshitz Transitions Induced by Field-Effect Doping in Strained $\mathrm{MoS}_{2}$ Nanolayers, J. Phys. Condens. Matter 31, 114002 (2019).

[82] S. Lebègue and O. Eriksson, Electronic Structure of TwoDimensional Crystals from Ab Initio Theory, Phys. Rev. B 79, 115409 (2009).
[83] Y. Nakata, K. Sugawara, S. Ichinokura, Y. Okada, T. Hitosugi, T. Koretsune, K. Ueno, S. Hasegawa, T. Takahashi, and T. Sato, Anisotropic Band Splitting in Monolayer $\mathrm{NbSe}_{2}$ : Implications for Superconductivity and Charge Density Wave, npj 2D Mat. Appl. 2, 12 (2018).

[84] J. M. An and W. E. Pickett, Superconductivity of $\mathrm{MgB}_{2}$ : Covalent Bonds Driven Metallic, Phys. Rev. Lett. 86, 4366 (2001).

[85] P. Giannozzi et al., QUANTUM ESPRESSO: A Modular and Open-Source Software Project for Quantum Simulations of Materials, J. Phys. Condens. Matter 21, 395502 (2009).

[86] P. Giannozzi et al., Advanced Capabilities for Materials Modelling with Quantum ESPRESSO, J. Phys. Condens. Matter 29, 465901 (2017).

[87] G. Prandini, A. Marrazzo, I. E. Castelli, N. Mounet, and N. Marzari, Precision and Efficiency in Solid-State Pseudopotential Calculations, npj Comput. Mater. 4, 72 (2018).

[88] M. van Setten, M. Giantomassi, E. Bousquet, M. Verstraete, D. Hamann, X. Gonze, and G.-M. Rignanese, The PseudoDojo: Training and Grading a 85 Element Optimized Norm-conserving Pseudopotential Table, Comput. Phys. Commun. 226, 39 (2018).

[89] A. Togo and I. Tanaka, First Principles Phonon Calculations in Materials Science, Scr. Mater. 108, 1 (2015). 